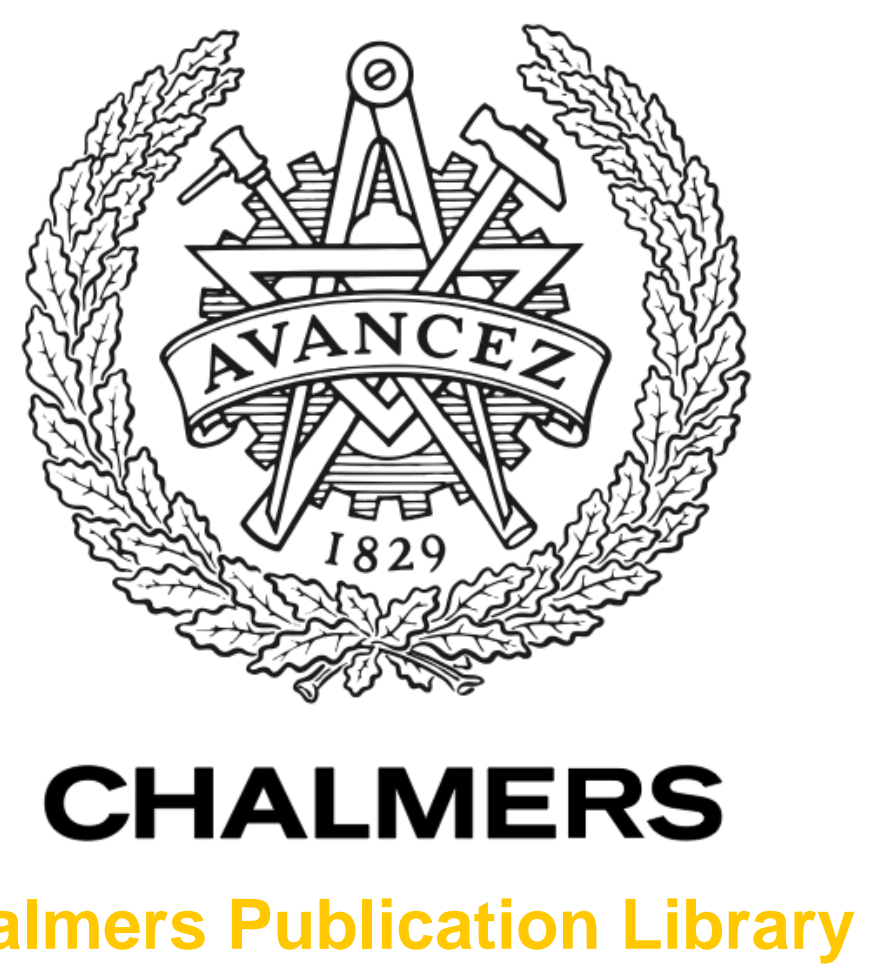

\title{
Toroidal modelling of RMP response in ASDEX Upgrade: coil phase scan, q(95) dependence, and toroidal torques
}

This document has been downloaded from Chalmers Publication Library (CPL). It is the author's version of a work that was accepted for publication in:

Nuclear Fusion (ISSN: 0029-5515)

Citation for the published paper:

Liu, Y. ; Ryan, D. ; Kirk, A. et al. (2016) "Toroidal modelling of RMP response in ASDEX Upgrade: coil phase scan, q(95) dependence, and toroidal torques". Nuclear Fusion, vol. $56(5)$

http://dx.doi.org/10.1088/0029-5515/56/5/056015

Downloaded from: http://publications.lib.chalmers.se/publication/237031

Notice: Changes introduced as a result of publishing processes such as copy-editing and formatting may not be reflected in this document. For a definitive version of this work, please refer to the published source. Please note that access to the published version might require a subscription. 


\title{
Toroidal modelling of RMP response in ASDEX Upgrade: coil phase scan, $q_{95}$ dependence, and toroidal torques
}

Yueqiang Liu ${ }^{1,2,3}$, D. Ryan ${ }^{4,1}$, A. Kirk ${ }^{1}, \mathrm{Li} \mathrm{Li}^{5,6}$, W. Suttrop ${ }^{7}$, M. Dunne ${ }^{7}$, R. Fischer ${ }^{7}$, J.C. Fuchs $^{7}$, B. Kurzan ${ }^{7}$, P. Piovesan ${ }^{8}$, M Willensdorfer ${ }^{7}$ and the ASDEX Upgrade team ${ }^{7}$ and the EUROfusion MST1 team ${ }^{9}$

${ }^{1}$ CCFE, Culham Science Centre, Abingdon, OX14 3DB, UK

2 Southwestern Institute of Physics, PO Box 432, Chengdu 610041, China

${ }^{3}$ Department of Earth and Space Science, Chalmers University of Technology, SE-412 96

Gothenburg, Sweden

${ }^{4}$ Department of Physics, University of York, Heslington, York, YO10 5DD, UK

${ }^{5}$ Donghua University, Shanghai, China

${ }^{6}$ Forschungszentrum Jülich GmbH, Institut för Energie-und Klimaforschung-Plasmaphysik, Jülich, Germany

${ }^{7}$ Max Planck Institute for Plasma Physics, Garching, Germany

${ }^{8}$ Consorzio RFX, Euratom-ENEA Association, Padova, Italy

${ }^{9}$ See http://www.euro-fusionscipub.org/mst1

E-mail: yueqiang.liu@ccfe.ac.uk

\begin{abstract}
The plasma response to the vacuum resonant magnetic perturbation (RMP) fields, produced by the ELM control coils in ASDEX Upgrade experiments, is computationally modelled using the MARS-F/K codes [Liu Y.Q. et al 2000 Phys. Plasmas 7 3681; Liu Y.Q. et al 2008 Phys. Plasmas 15 112503]. A systematic investigation is carried out, considering various plasma and coil configurations as in the ELM control experiments. The low $q$ plasmas, with $q_{95} \sim 3.8$ ( $q_{95}$ is the safety factor $q$ value at $95 \%$ of the equilibrium poloidal flux), responding to low $n$ ( $n$ is the toroidal mode number) field perturbations from each single row of the ELM coils, generates a core kink amplification effect. Combining two rows, with different toroidal phasing, thus leads to either cancellation or reinforcement of the core kink response, which in turn determines the poloidal location of the peak plasma surface displacement. The core kink response is typically weak for the $n=4$ coil configuration at low $q$, or for the $n=2$ configuration but at high $q\left(q_{95} \sim 5.5\right)$. A phase shift of around 60 degrees for low $q$ plasmas, and around 90 degrees for high $q$ plasmas, is found in the coil phasing, between the plasma response field and the vacuum RMP field, that maximizes the edge resonant field component. This leads to an optimal coil phasing of about 100 (-100) degrees for low (high) $q$ plasmas, that maximizes both the edge resonant field component and the plasma surface displacement near the X-point of the separatrix. A strong parallel sound wave damping moderately reduces the core kink response but has minor effect on the edge peeling response. For low $q$ plasmas, modelling shows that both the resonant electromagnetic torque and the neoclassical toroidal viscous (NTV) torque (due to the presence of 3D magnetic field perturbations) contribute to the toroidal flow damping, in particular near the plasma edge region. For high $q$ plasmas, however, significant amount of torque is also produced in the bulk plasma region, and the contributions from the electromagnetic, the NTV, and the torque associated with the Reynolds stress, all play significant roles.
\end{abstract}




\section{Introduction}

It is well realized that large scale edge localized modes (ELMs), mainly the so called type-I ELMs, may pose a danger of intolerable material damage in future large fusion devices such as ITER [1]. Various techniques have been envisaged for the purpose of mitigating or suppressing type-I ELMs, such as the pellet pacing [2], the vertical kicking of the plasma position [3], and finally the application of resonant magnetic perturbation (RMP) fields [4]. So far RMPs are probably the most exploited technique, having been applied for ELM control on many present day tokamak devices where $\mathrm{H}$-mode plasmas are achieved [5].

Understanding ELM control with RMP may involve various levels of physics. At the macroscopic level, the plasma response to the three-dimensional (3D) RMP fields plays an important role [6]. This essentially involves three aspects. First, the plasma response modifies the poloidal spectrum of the applied vacuum field, often manifested by two effects - the screening of the magnetic field pitch-aligned resonant poloidal components near rational surfaces $[7,8,6,9]$, and the amplification of certain non-resonant components $[10,11,12]$. Both effects can be substantial depending on the plasma and the RMP field configurations, and can even be inherently coupled [12]. Secondly, the plasma response leads to 3D deformation of an otherwise 2D axi-symmetric tokamak equilibrium, eventually forming a 3D equilibrium $[13,14]$. This may have several consequences, in particular, in terms of the ELM control physics, on the stability of the underlying peeling-ballooning modes [15]. Finally, toroidal torques, of various physics origins, can be generated as a result of the plasma response, that may in turn act on the plasma by changing the toroidal flow $[16,17,18]$.

Several families of plasma response models have been considered in literatures during recent years. In terms of 3D physics, either the so called 3D perturbed equilibrium approach $[19,6]$, or a full 3D equilibrium approach [20, 21, 22, 23], has been followed. In the former, the plasma response is treated as a linear perturbation to the $2 \mathrm{D}$ equilibrium. The basic assumption here is that the amplitude of the applied 3D RMP field is much smaller (often by several orders of magnitude) than the equilibrium field. More subtle requirements may also be imposed on the perturbative approach, both near rational surfaces as well as in terms of certain geometric constraints for the plasma displacement [24]. The full 3D equilibrium approach assumes that a full force balance condition is satisfied by the plasma response. This seemingly simple and accurate requirement may actually involve non-trivial treatments near rational surfaces, where singularities occur in the ideal magnetohydrodynamic (MHD) assumption, or where magnetic reconnection takes place for a resistive plasma. In fact the ideal versus resistive plasma response can be viewed as another way of distinguishing the plasma response models. Inertial forces due to plasma flow further complicate the 3D force balance [25].

The other family of plasma response model is formed by considering a cascade of physics that may be relevant to the RMP problem, ranging from the single fluid approximation $[19,6]$, the two fluid effects which essentially involve the diamagnetic flow corrections $[9,26]$, to detailed kinetic physics $[27,28]$ which may indeed be critical for describing the pedestal region with sharply varying spacial and temporal scales. Here again linear, quasi-linear, or fully non-linear approximations can be made for various physics combinations.

The single fluid, resistive model in the linear approximation, which is what we adopt in this work, may seem crude for studying the RMP problem. On the other hand, it captures many of 
the basic physics effects, such as the flow induced field screening, the plasma response induced magnetic field amplification, the 3D distortion of the plasma surface, and finally a lowest order estimate on the momentum confinement due to external 3D fields. Perhaps more importantly, this model has been extensively demonstrated to be capable of quantitatively describing the measured RMP response in experiments [29, 11, 30, 28]. Another obvious advantage of the linear single fluid model is the computational efficiency, which easily allows (i) full MHD (i.e. no reduced MHD formulation as is often adopted in non-linear models) treatment in full toroidal geometry, and (ii) systematic investigation of the aforementioned physics affects for multiple equilibrium and coil configurations. In addition, the linearity of the problem allows us to numerically accurately model plasmas with realistic Lundquist numbers.

In this work, we carry out a systematic modelling of the plasma response for the recent RMP experiments in ASDEX Upgrade, mainly using the MARS-F code [31]. These experiments were performed for low collisionality plasmas [32]. We also compare the modelling results with experimental observations [32], although the comparison is not comprehensive. In the ASDEX Upgrade RMP experiments, two rows of magnetic coils (called B-coils), each consisting of 8 window-frame coils distributed along the toroidal angle of the torus, are located inside the vacuum vessel, above and below the outboard mid-plane, respectively, and referred to as the upper and lower coils in this work. The coils are powered up to $6.5 \mathrm{kAt}$ current. In the majority of the discharges, the coils are configured to produce a dominant $n=2$ toroidal component of the vacuum field, though RMP experiments have also been carried out with the $n=1$ and $n=4$ coil configurations [32]. The coil currents can be powered in the presence of a toroidal phase shift between the upper and lower rows, sometimes referred to as toroidal coil phasing in further discussions. This toroidal phase can be continuously scanned in experiments with the $n=2$ configuration. In the ELM control experiments in ASDEX Upgrade, two typical plasma configurations are considered, differing by the edge safety factor $q_{95}$. The low- $q$ (high- $q$ ) discharges have typical $q_{95}$ values around 3.8 (5.5).

We shall compute the plasma response for a set of equilibria from different discharges. Several aspects of the plasma response will be examined, including the poloidal spectrum of the response radial field compared to that of the vacuum field, the suppression/amplification of the pitch-resonant versus non-resonant components, the plasma internal displacement associated with the core kink versus the edge peeling components of the response, the plasma surface displacement near the low field side mid-plane and near the X-point. The ultimate purpose of such a systematic investigation is to identify, on the macroscopic level, indicators that correlate to experimental observations (e.g. the change of the ELM frequency, the density pump out) during the ELM control, thus providing guidance for planning future experiments in ASDEX Upgrade. The modelling results are also compared with that in other devices, in particular in MAST [10], seeking for commonalities. Finally, toroidal torques will be computed and compared among various plasma-coil configurations, for the purpose of qualitative interpretation of the toroidal flow damping observed in some of the recent ASDEX Upgrade RMP experiments.

The next Section defines details of the plasma-coil configurations chosen in this study, followed by a brief description of the computational models in Section 3. Section 4 reports the main computational results, covering a list of important aspects directly relevant to experiments, including the effects of toroidal phasing of coil currents, the role of parallel sound wave damping on the plasma response, the effect of $q_{95}$, as well as a comparison between the 
Table 1: Basic equilibrium parameters of the modelled plasmas.

\begin{tabular}{|c|c|c|c|c|c|c|c|c|}
\hline Shot\# & Time $(\mathrm{ms})$ & $R_{0}(\mathrm{~m})$ & $B_{0}(\mathrm{~T})$ & $I_{p}(\mathrm{MA})$ & $\beta_{N}$ & $q_{0}$ & $q_{95}$ & $\Omega_{0} / \omega_{A}(\%)$ \\
\hline \hline 28061 & 1615 & 1.650 & 1.730 & 0.795 & 2.013 & 1.244 & 3.708 & 0.683 \\
\hline 30835 & 3200 & 1.724 & 1.705 & 0.773 & 2.148 & 0.811 & 3.760 & 2.299 \\
\hline 31128 & 3500 & 1.706 & 1.790 & 0.766 & 1.669 & 0.900 & 3.894 & 2.504 \\
\hline 30684 & 4005 & 1.717 & 2.380 & 0.780 & 1.479 & 1.179 & 5.533 & 2.893 \\
\hline 30684 & 6950 & 1.712 & 2.389 & 0.783 & 1.503 & 1.206 & 5.506 & 2.350 \\
\hline 31131 & 6400 & 1.736 & 1.739 & 0.537 & 2.175 & 1.145 & 5.563 & 3.348 \\
\hline
\end{tabular}

$n=2$ versus $n=4$ coil configurations. A parallel is drawn with certain key experimental observations. Section 5 reports computational results of toroidal torques for various plasma-coil configurations. These results are then applied for a qualitative interpretation of the observed rotation braking in experiments. Section 6 draws conclusions.

\section{Plasma and coil configurations}

Table 1 lists all the representative ASDEX Upgrade discharges that we have modelled in this work, together with the key equilibrium parameters, where $R_{0}$ is the plasma major radius, $B_{0}$ the toroidal vacuum field at $R_{0}, I_{p}$ the total plasma current, $\beta_{N} \equiv \beta(\%) a(\mathrm{~m}) B_{0}(\mathrm{~T}) / I_{p}(\mathrm{MA})$, with $\beta$ being the plasma volume averaged pressure normalized by the magnetic pressure, and $a$ the plasma minor radius. Listed in the Table are also the values of the safety factor $q$ on the magnetic axis $\left(q_{0}\right)$ and at the $95 \%$ equilibrium poloidal flux surface $\left(q_{95}\right)$, as well as experimentally measured on-axis toroidal rotation frequency $\Omega_{0}$ normalized by the on-axis toroidal Alfvén frequency $\omega_{A}$. Unless otherwise stated, the measured rotation (both amplitude and radial profile) is used as the input for the MARS-F modelling. The rotation amplitude does not significantly vary in these experiments, except in an earlier discharge 28061.

Note that for discharge 30684, we reconstruct the plasma equilibria at two different time slices. This is largely for the purpose of sensitivity study of the computational results against the variation of the plasma during the discharge. Also note that these discharges cover two different $q$ profiles, with $q_{95}$ around 3.8 and 5.5, respectively.

These plasma equilibria are reconstructed by the CLISTE code [33] based on the experimental measurements. Another fixed-boundary equilibrium code CHEASE [34] is subsequently used, based on the CLISTE output, in order to re-produce, refine the equilibria, and to generate computational input data for further MARS-F runs.

Figure 1 shows one example of the plasma boundary, for the equilibrium reconstructed from the discharge 31131 at $6400 \mathrm{~ms}$. Note that the original plasma boundary, from the CLISTE reconstruction, has an X-point associated with the separatrix surface. The X-point is slightly smoothed during the CHEASE reconstruction, in order to improve computational accuracy. This procedure does not significantly affect the computational results for the ASDEX Upgrade plasmas, as shown in Ref. [12]. 


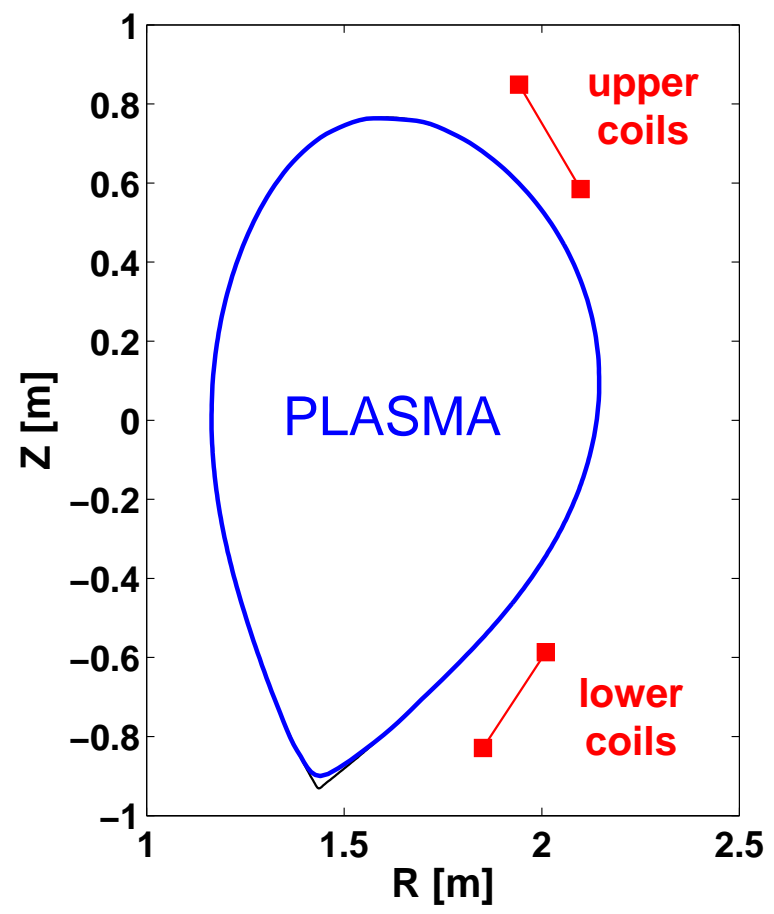

Figure 1: The plasma boundary shape reconstructed for discharge 31131 at $6400 \mathrm{~ms}$, and the locations of the RMP coils on the $(R, Z)$-plane.

Figure 1 also shows the location of the RMP coils in the $(R, Z)$-plane. As mentioned before, a $2 \times 8$ in-vessel coil system is utilized in ASDEX Upgrade. The coils are configured to produce either the predominant $n=2$ or the $n=4$ field perturbations. For the $n=2$ configuration, the coils are powered in such a way, that the toroidal phasing between the upper and lower rows can be continuously varied during the discharge. Either odd or even parity is possible in the $n=4$ configuration. In the MARS-F modelling, we assume an analytic $\exp (-i n \phi)$ variation of the coil current along the toroidal angle $\phi$. The amplitude of the coil current is calculated by Fourier decomposing the experimental coil current distribution along the toroidal angle, and taking the $n$-th component. We note that 8 coils along the toroidal angle in each row also produce sideband harmonics. For example, with the $n=2$ coil configuration, the sidebands are $n=10,18, \ldots,-6,-14, \ldots$. Given the toroidal coverage of each B-coil in ASDEX Upgrade, the ratios of these sideband current amplitude, to that of the main component $n=2$, are estimated as $15 \%, 5 \%, \ldots, 41 \%, 20 \%, \ldots$, respectively. Since these sidebands are of rather high harmonic numbers, their effect on the plasma is probably weak.

\section{Computational models}

MARS-F solves perturbed full resistive MHD equations in the single fluid approximation, in full toroidal geometry. A pure vacuum region surrounding the plasma is also assumed in the model, with the vacuum equations (basically curl- and divergence-free conditions) for the perturbed magnetic field being solved in toroidal geometry, together with the MHD equations in the region occupied by the plasma. Passive conducting structures such as resistive walls 
(typically the vacuum vessel), the RMP coils, are also modelled, with the corresponding electromagnetic equations self-consistently solved with the aforementioned equations. Detailed MARS-F formulation with respect to the RMP application can be found in Ref. [6]. Below we discuss specific aspects/models that are directly relevant to the present work.

First we emphasize that, in terms of overall mathematical formulation, we solve a driven system, where the source terms are the currents flowing in the RMP coils. Therefore, even though the system of equations is linear, there is a steady state solution (physically the plasma response), whose amplitude is proportional to that of the RMP currents. This is different from a linear eigenvalue problem, where the amplitude of the solution (the eigenfunction) is not determined. Note that in the plasma response computations, we assume that the plasma is linearly stable to the given $n$ numbers ( $n=2$ or 4 in this study), which is normally the case. On the other hand, the plasma can be unstable to higher $n$ number modes which are directly responsible for the onset of type-I ELMs in these H-mode discharges. But we are not computing the plasma response of these high $n$ modes.

The presence of time varying RMP field also induces eddy currents in resistive walls such as the vacuum vessel or the conducting plates in ASDEX Upgrade. However, in experiments where the RMP coils are powered with dc-currents (or ac-currents but varying at much longer time scale than the penetration time of the conducting structures), and where we are interested in the steady state response, the conducting structures do not play a role, and therefore are not included into the model. These are the cases considered in the present study.

The Spitzer model is used for the plasma resistivity $\eta$ in this work. The radial profile of the resistivity is thus assumed to be proportional to $T_{e}^{-3 / 2}$, where $T_{e}$ is the thermal electron temperature as measured in experiments. The amplitude of the resistivity enters into the MARS-F equations via the on-axis Lundquist number $S \equiv \tau_{R} / \tau_{A}$, where $\tau_{R} \equiv \mu_{0} a^{2} / \eta_{0}$ and $\tau_{A} \equiv R_{0} / v_{A}$. $\mu_{0}$ is the vacuum permeability. Both the resistivity $\eta_{0}$ and the toroidal Alfvén speed $v_{A}$ here are evaluated at the magnetic axis. For the ASDEX Upgrade plasmas studied in this work, the on-axis value of $S$ varies between $1.1 \times 10^{8}$ and $3.4 \times 10^{8}$, according to the Spitzer model.

Next we discuss the flow model. The equilibrium toroidal flow is included into the MARS-F MHD equations. Both amplitude and the radial profile of the flow speed are specified as the input data to the code. In particular, the angular frequency of the toroidal rotation, from the experimental measurements, is specified as a function of the equilibrium poloidal flux. The amplitude of the on-axis rotation frequency is reported in Tab. 1. In the RMP problem, the toroidal flow essentially plays a role of screening the pitch-resonant components of the externally applied 3D magnetic field perturbation, as a result of the plasma response. A detailed discussion of this aspect, valid for a generic toroidal geometry, can be found in Appendix of Ref. [35].

Finally, a parallel sound wave damping (SWD) model is utilized in the MARS-F formulation, via an equivalent viscous force

$$
\nabla \cdot \Pi=\rho \kappa_{\|}\left|k_{\|} v_{t h, i}\right| \mathbf{v}_{\|},
$$

acting as a sink term in the perturbed momentum balance equation. Here $\rho$ is the equilibrium plasma density, $\kappa_{\|}$is a numerical coefficient specifying the "strength" of the damping. $k_{\|}=(n-m / q) / R_{0}$ is the parallel wave number, with $m$ being the poloidal harmonic number. $v_{t h, i}=\sqrt{2 T_{i} / M_{i}}$ is the thermal ion velocity, with $T_{i}, M_{i}$ being the thermal ion temperature and 
mass, respectively. Note that this damping term only acts on the parallel component of the momentum equation. Physically, this model describes the Landau damping of the parallel sound waves due to the ion-acoustic resonances [36]. Thus this model can be viewed as a kinetic correction to the standard MHD model, specifically along the parallel motion, where the MHD description is poor in the presence of equilibrium flow [37].

Since the parallel sound wave damping model allows a numerically adjustable coefficient $\kappa_{\|}$, in this work we shall investigate the sensitivity of the computed plasma response against the choice of $\kappa_{\|}$, by considering a weak SWD limit $\left(\kappa_{\|}=0.1\right)$ and a strong SWD limit $\left(\kappa_{\|}=1.5\right)$. Previous modelling results for other devices [29] indicate that the plasma response is not sensitive to the choice of SWD in low- $\beta$ plasmas, whilst the strong SWD better describes the experimental results [39] in higher- $\beta$ plasmas. When the plasma pressure approaches (or even exceeds) the no-wall beta limit for the ideal kink mode, comparison between modelling and DIII-D experiments [28] shows that a full drift kinetic model, such as the MARS-K model [40], may be necessary. The MARS-K model recovers the fluid response results at low beta. For the ASDEX Upgrade plasmas studied here, the normalized beta value is relatively high (see Tab. 1), but is still below the no-wall beta limit. Therefore, we expect the SWD model may provide reasonable results.

The MARS-F code has been subject to various benchmarking against other codes [24]. The code is also validated by comparing the RMP modelling results with experiments in DIIID [38, 28] and MAST [41]. For the quantitative RMP modelling, it is also important to accurately model the vacuum field generated by the coil currents. Figure 2 shows one example of comparison for the ASDEX Upgrade discharge 28061, where both MARS-F and the BiotSavart law based ERGOS code [42] are used to compute the amplitude of the pitch-resonant vacuum radial field, at the corresponding rational surfaces. The agreement is good. Here the radial field component $b_{\text {res }}^{r}$ is defined as a dimensionless quantity

$$
b_{\mathrm{res}}^{r}=\frac{2}{R_{0}|\nabla s|_{m=0}}\left(\frac{\mathbf{b} \cdot \nabla s}{\mathbf{B}_{\mathrm{eq}} \cdot \nabla \phi}\right)_{m n},
$$

where $\mathbf{b}$ is the perturbed field, and $s \equiv\left[\left(\psi-\psi_{\text {axis }}\right) /\left(\psi_{\text {edge }}-\psi_{\text {axis }}\right)\right]^{1 / 2}$ is the normalized radial coordinate labelling equilibrium magnetic flux surfaces. $|\nabla s|_{m=0}$ is the $m=0$ poloidal Fourier harmonic of the equilibrium quantity $|\nabla s|$. The Fourier decomposition is performed in a straight field line (SFL) flux coordinate system.

\section{Plasma response computations}

The MARS-F toroidal modelling results reported below, as the result of a systematic investigation of the plasma response for ASDEX Upgrade, cover several important aspects.

\subsection{Cancellation of plasma response to upper and lower coils}

We start by comparing the plasma response to each row of the RMP coils separately, assuming that the two rows of coils are independently powered. Besides the interesting physics insight 


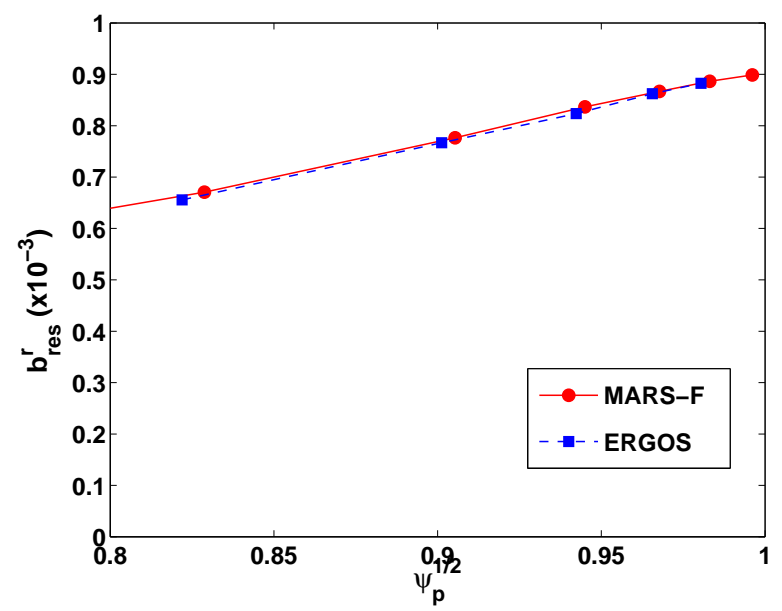

Figure 2: Comparison of the amplitude of the pitch-aligned components of the vacuum field, computed by MARS-F and ERGOS, respectively, for the discharge 28061 at $1615 \mathrm{~ms}$, with the $n=2$ coil configuration at even parity.

that can be obtained from such a study, the results also help to understand the coil phasing scan results to be discussed later on. We mention that in the ASDEX Upgrade experiments modelled in this work, a special arrangement of the coil power supply is made [32], which does not allow independent activation of the upper and lower rows.

Figure 3 compares the dominant poloidal Fourier harmonics $(m / n=2 / 2$ and $3 / 2$, in SFL coordinates) of radial field, produced by the upper and lower rows separately. The coil currents have the same amplitude and phase for both rows. The radial field $b^{1}$ here is again defined as a dimensionless quantity and decomposed in poloidal (and toroidal) Fourier harmonics as $b^{1}=\sum b_{m}^{1} \exp (i * m \chi-i * n \phi)$, where $\chi$ is the SFL poloidal angle, $n=2$ in this case, and

$$
b_{m}^{1}=\frac{q}{R_{0}^{2} B_{0}}\left(\frac{\mathbf{b} \cdot \nabla \psi}{\mathbf{B}_{\mathrm{eq}} \cdot \nabla \phi}\right)_{m n} .
$$

Both real and imaginary parts of $b_{m}^{1}$ are compared. In real space, these two parts represent the field perturbations that are toroidally separated by $45^{\circ}$ (for $n=2$ ).

The vacuum field is compared to the total field including the plasma response, showing two major effects. The most evident one is the strong amplification of the vacuum field components, due to the plasma response, to a single row of coils, inside the respective rational surfaces ( $q=1$ for the $2 / 2$ component and $q=1.5$ for the $3 / 2$ component). This is now often referred to as the core kink amplification. Note that the core kink amplification is not directly related to the fact that $q_{0}$ is below 1 in this equilibrium, since similar amplification is also observed for the $m / n=3 / 2$ harmonic. Moreoever, modelling of other equilibria with $q_{\min }$ above 1 shows similar core kink amplification effect.

However, whether this amplification actually occurs when both rows of coils are powered, depends on the choice of the relative toroidal phasing of the coil currents between the upper and lower rows. If the coil currents are in-phase (even parity), cancellation occurs as shown in Fig. 3. As a result, no core kink amplification occurs for this configuration. If the coil currents are anti-phase (odd parity), strong core kink amplification, with respect to vacuum, occurs 

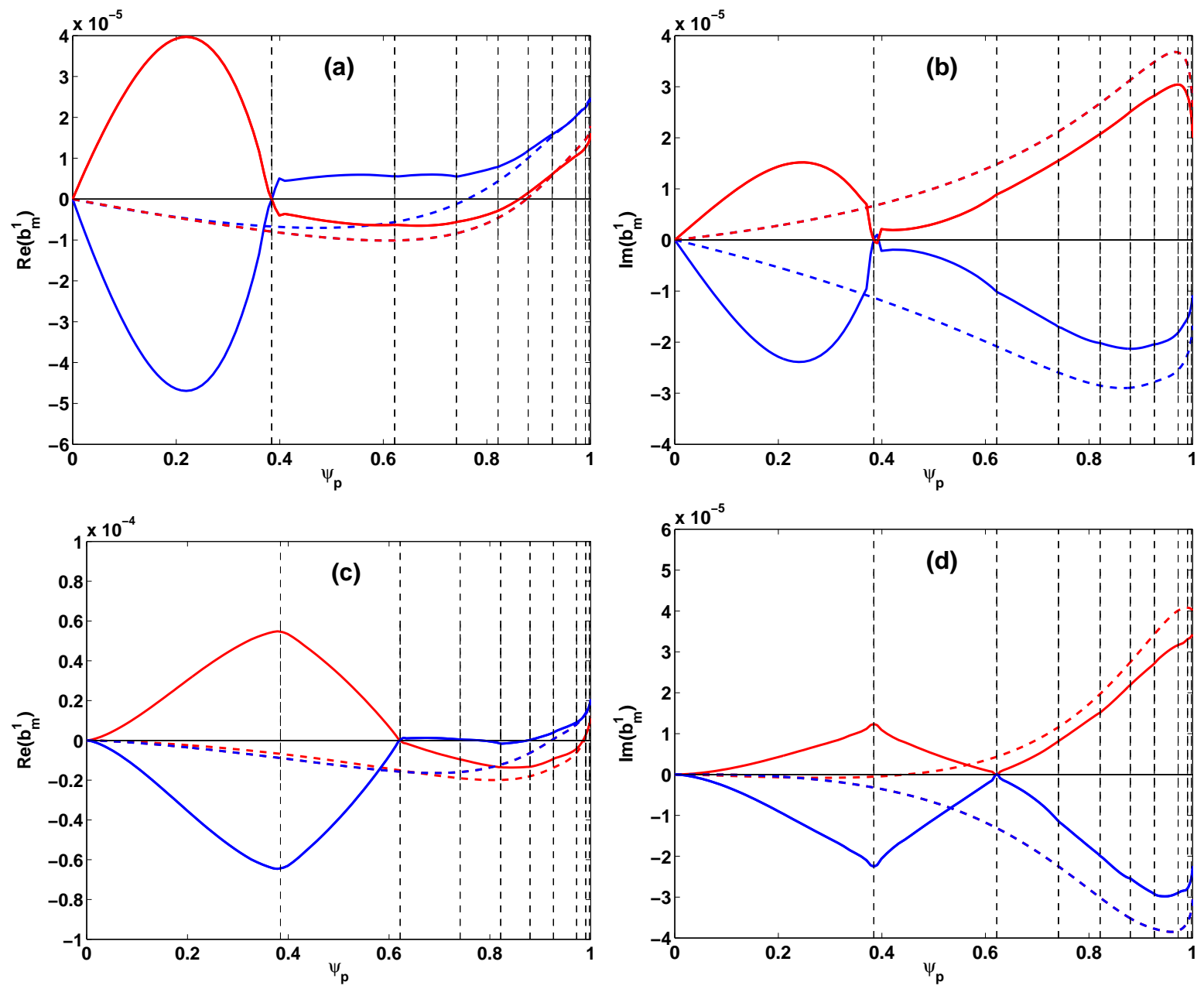

Figure 3: Plasma response field (solid) compared with the vacuum field (dashed), for (a-b) the $m / n=2 / 2$ and (c-d) the $m / n=3 / 2$ harmonics, based on discharge 30835 at $3200 \mathrm{~ms}$. Both $(\mathrm{a}, \mathrm{c})$ real and $(\mathrm{b}, \mathrm{d})$ imaginary parts of the Fourier components are shown. The field from the upper coils alone (blue) is compared with that from the lower coils alone (red), with zero toroidal phase for the coil current in both cases. Vertical dashed lines indicate the radial location of rational surfaces. 

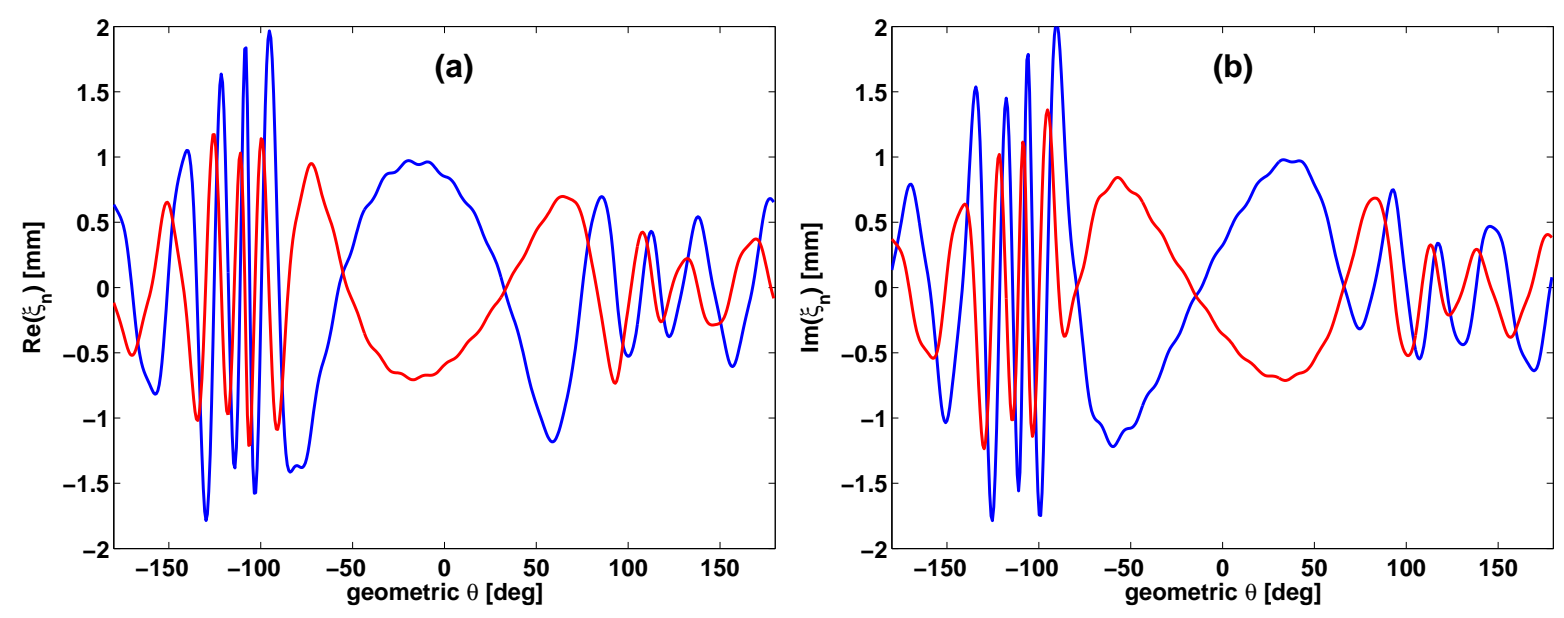

Figure 4: The (a) real, and (b) imaginary, parts of the MARS-F computed plasma surface displacement along the geometric poloidal angle, for discharge 30835 at 3200ms with the $n=2$ RMP field configuration. Compared are two cases where the upper coils alone (blue) and lower coils alone (red) are powered, with zero toroidal phasing for the coil current in both cases.

for the real part of the response field components (both $2 / 2$ and 3/2), as a result of changing the field parity by the plasma response. The results shown here thus indicate that the plasma amplification effect crucially depends on the coil configuration - the inherent amplification may occur to the field produced by each individual row of coils, but may not eventually appear due to the cancellation effect.

The choices of odd and even parities present two extreme cases in this plasma-coil configuration. With the continuous variation of the toroidal phasing between the two rows, we obtain partial cancellation or amplification.

Associated with the magnetic field response is also the 3D plasma surface displacement. Figure 4 compares the computed normal displacement amplitude of the plasma surface along the geometric poloidal angle $\theta$, for the same plasma-coil setup as in Fig. 3. Parity analysis shows a strong cancellation of the plasma displacement near the outboard mid-plane $\left(\theta \sim 0^{\circ}\right)$, if the upper and lower row coil currents have the same toroidal phase, leading to a large plasma displacement peaking near the X-point $\left(\theta \sim-110^{\circ}\right)$. On the contrary, the odd parity coil configuration reinforces the mid-plane displacement.

Relating the plasma surface displacement to the field response shown in Fig. 3, we find a correlation between the core kink amplification and the outboard mid-plane displacement peaking. On the other hand, the cancellation of the core kink amplification leads to the predominance of the edge peeling-tearing response, which in turn correlates to the plasma displacement peaking near the X-point. Similar correlations have been found for MAST plasmas [10]. Further on, we shall exploit the robustness of this correlation for other ASDEX Upgrade plasmas in the $n=2$ RMP configuration.

The aforementioned cancellation/reinforcement effect due to the $n=2$ coil parity is also found with the $n=1$ coil configuration in the MARS-F modelling. An example is shown in Figure 5, for the same ASDEX Upgrade equilibrium as in Figs. 3-4. Opposite to that of the $n=2$ 

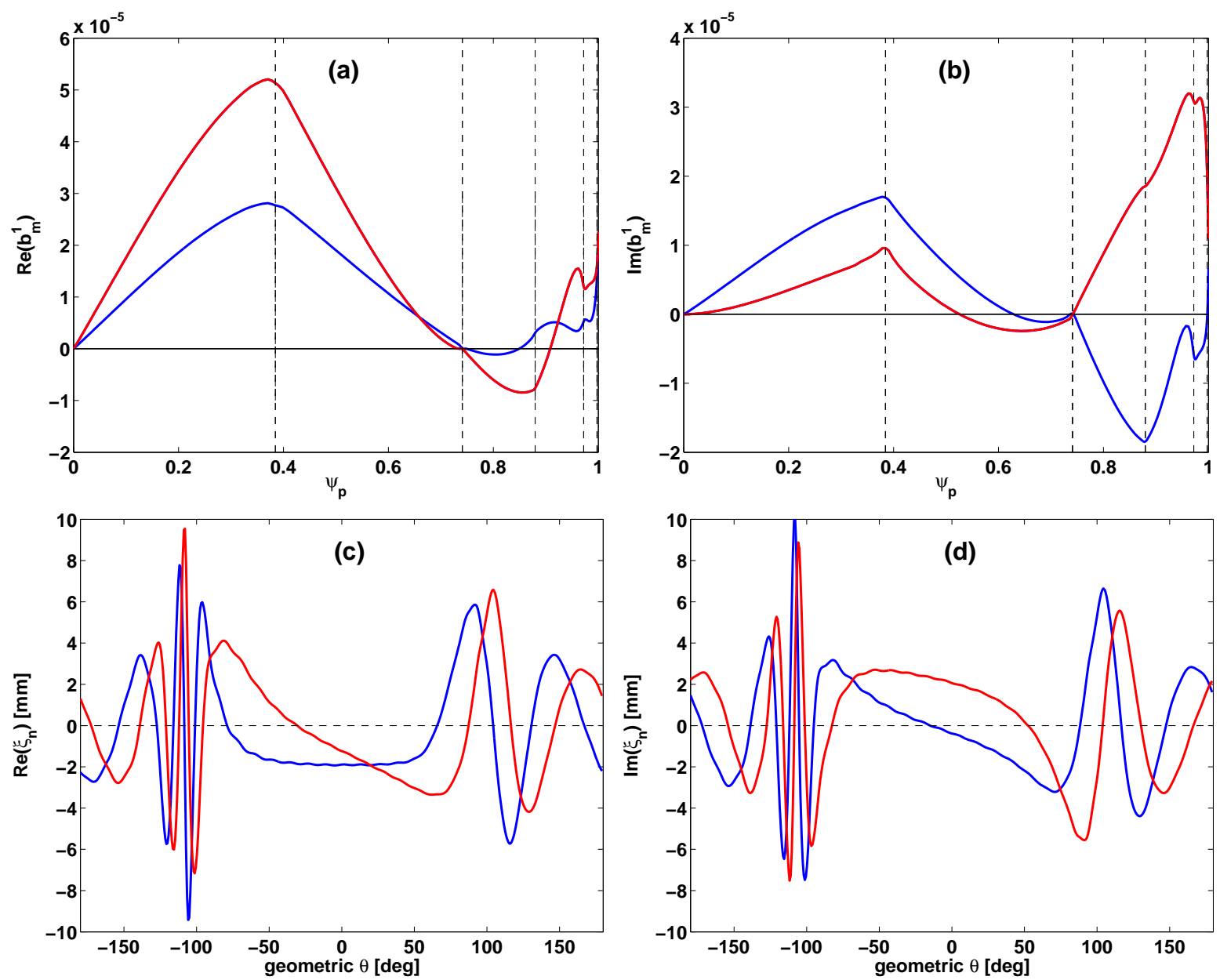

Figure 5: Comparison of (a) the real, and (b) the imaginary, parts of the the $m / n=2 / 1$ harmonics of the plasma response field, and (c) the real, (d) the imaginary, parts of the plasma surface displacement, based on discharge 30835 at 3200ms with the $n=1$ RMP configuration. The results from the upper coils alone (blue) is compared with that from the lower coils alone (red), with zero toroidal phasing for the coil current in both cases. Vertical dashed lines in (a) and (b) indicate the radial location of rational surfaces.

configuration, the $n=1$ even coil parity reinforces the core kink field response as well as the low field side mid-plane plasma displacement as shown in the figure. Consequently, the $n=1$ odd coil parity will cancel the core kink response as well as enhance the X-point displacement. Nevertheless, the correlations between the core kink (edge peeling) field response and the midplane (X-point) displacement also holds for the $n=1$ configuration.

\subsection{Suppression of core plasma response by parallel sound wave damp- ing}

The results presented in the previous subsection are obtained assuming a weak parallel SWD model with $\kappa_{\|}=0.1$. As discussed before, the parallel SWD does not significantly affect the response of a low- $\beta$ plasma. Since the ASDEX Upgrade plasmas in the RMP experiments 
have finite $\beta$, we wish to understand how this damping physics can affect the plasma response. First of all, we have checked that the SWD model does not qualitatively affect the parity modifications, and consequently the cancellation/reinforcement effects discussed in the previous subsection. However, it does quantitatively affect the core plasma response.

We show this for another ASDEX Upgrade discharge in the following figures 6 and 7. Similar effects are found with other ASDEX Upgrade discharges.

Figure 6 compares the poloidal spectrum of the radial fields including the plasma response, from the MARS-F computations with weak (top panel) and strong (bottom panel) SWD. Three toroidal phasing of the RMP coil currents are considered, with $\Delta \Phi=0^{\circ}$ (even parity), $\Delta \Phi=$ $90^{\circ}$, and $\Delta \Phi=180^{\circ}$ (odd parity), respectively. Here $\Delta \Phi \equiv \Phi^{\text {upper }}-\Phi^{\text {lower }}$ is defined as the phase difference between the upper and lower rows of the coil currents. The amplitude of the poloidal harmonics, as defined in (2), is plotted for all poloidal mode numbers, as well as along the normalized plasma minor radius $s=\sqrt{\psi_{p}}$, with $\psi_{p}$ being the normalized equilibrium poloidal flux. The locations of the rational surfaces (with $n=2$ ) are indicated by '+' symbols in these $2 \mathrm{D}$ plots.

First, we observe the usual screening of the pitch-resonant harmonics near the corresponding rational surfaces, due to the plasma response, resulting in the formation of a valley along the location of rationals surfaces indicated by ' + ' symbols. This screening effect is not modified by the strength of the SWD. On the other hand, the non-resonant part of the spectrum, in particular the core kink part (with $s \lesssim 0.5$ ), is generally reduced by the strong SWD. This is particularly evident at $\Delta \Phi=90^{\circ}$ and $180^{\circ}$. The largest core kink amplification occurs with the odd parity coil configuration (agreeing with Fig. 3) and with a weak SWD. The other parts of the spectrum is almost not affected by the damping model.

The damping of the core plasma response due to a strong SWD is also evident in the computed internal plasma displacement, shown in Fig. 7, where the amplitude of the poloidal Fourier harmonics for the radial displacement $\xi^{1} \equiv \xi \cdot \nabla s$ is compared. The core kink response, represented by harmonics $m=1-4$, is reduced with the strong SWD, but the edge peeling-tearing response, represented by harmonics with $m>10$, is almost not affected by the SWD.

In the corresponding experiments, there seems to be no large core plasma displacement that has been observed. This indication favours the strong SWD physics for modelling these ASDEX Upgrade plasmas.

\subsection{Plasma response versus toroidal phasing of coil currents}

We have so far been showing largely qualitative features of the plasma response. More quantitative comparison with parametric scans requires definition of relevant figures of merit. In this work, we define four sets of figures of merit. The first is the amplitude of the pitch-resonant radial field component nearest to the last closed flux surface, corresponding to the largest rational $q$ value, for a given $n$, within the plasma. This quantity, further denoted by $b_{\text {res }}^{1}$, characterizes the plasma edge screening due to the resistive plasma response. The magnitude of $b_{\text {res }}^{1}$ also determines the size of the magnetic island near the plasma edge. The second set consists of the amplitudes of the normal displacement $\xi_{n}$ of the plasma surface, defined at the outboard 

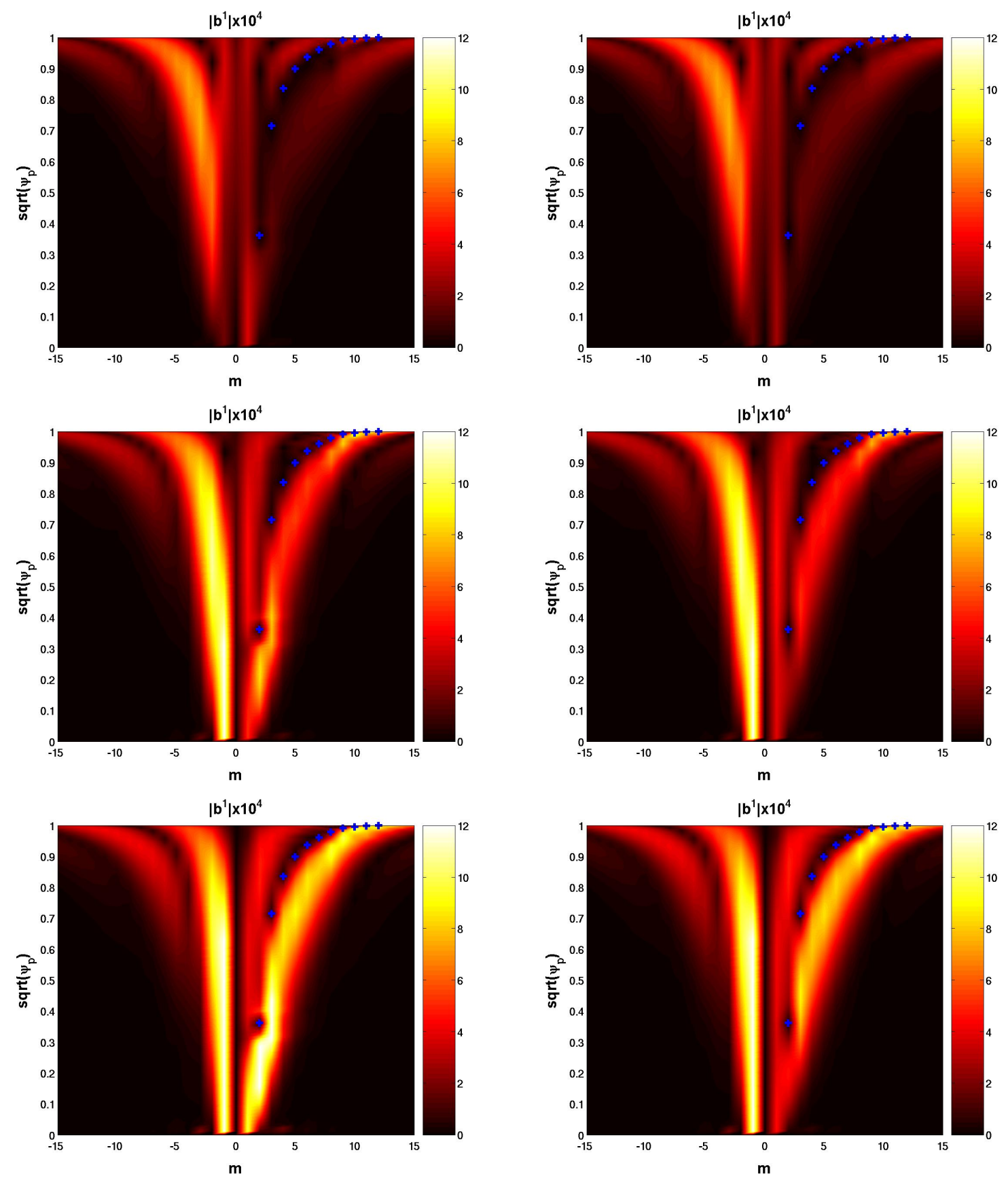

Figure 6: Poloidal spectrum of the computed plasma response radial field with weak (left panel) and strong (right panel) sound wave damping model, for the toroidal phasing of the coil currents $\Delta \Phi=0^{\circ}$ (a,d), $\Delta \Phi=90^{\circ}$ (b,e), and $\Delta \Phi=180^{\circ}$ (c,f), respectively. Modelling is based on an equilibrium from the low $q_{95}$ discharge 31128 at $3500 \mathrm{~ms}$, with the $n=2$ coil configuration. 

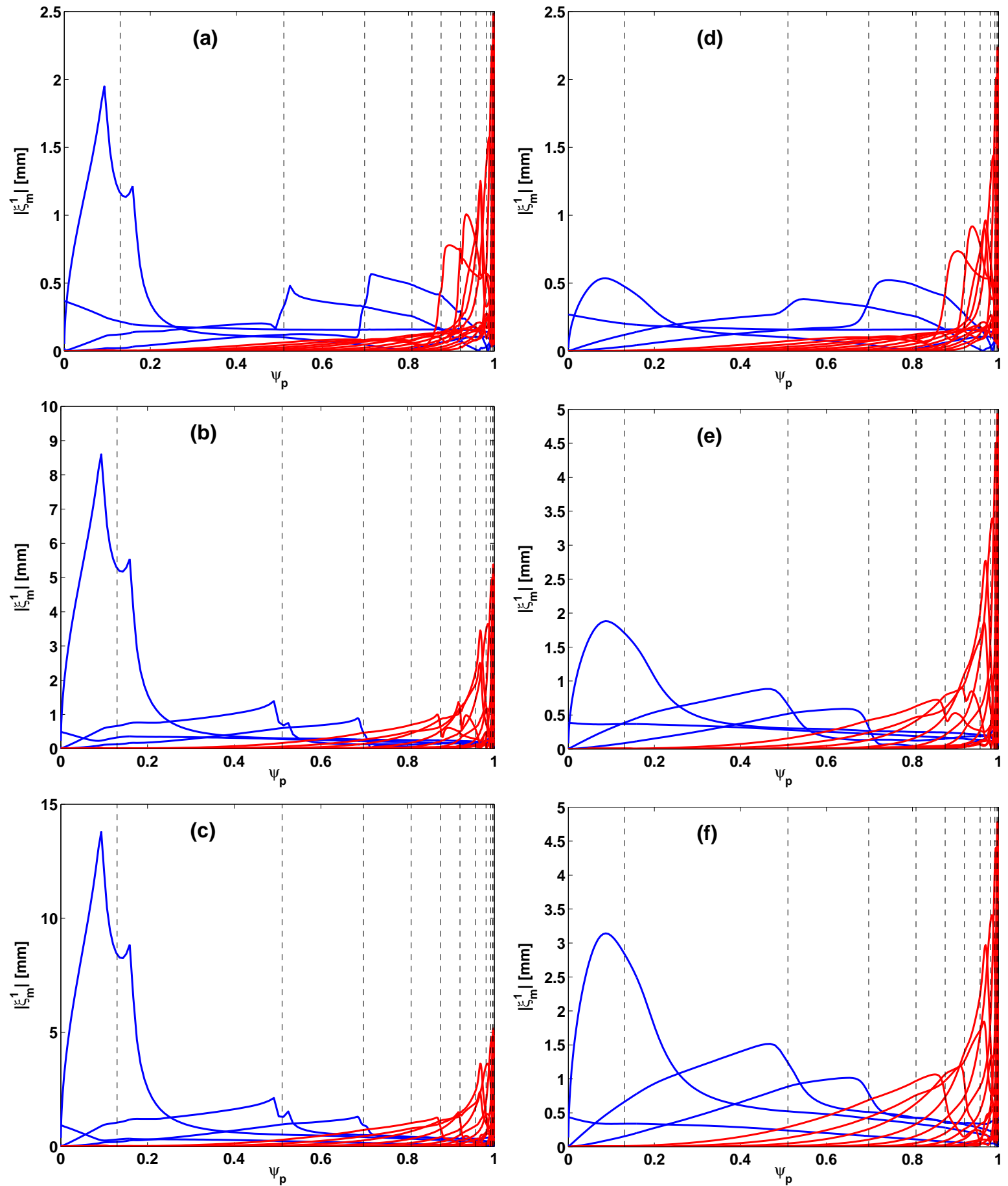

Figure 7: Poloidal Fourier harmonics of the computed radial displacement of the plasma, with weak (left panel) and strong (right panel) sound wave damping model, for the toroidal phasing of the coil currents $\Delta \Phi=0^{\circ}$ (a,d), $\Delta \Phi=90^{\circ}$ (b,e), and $\Delta \Phi=180^{\circ}$ (c,f), respectively. Modelling is based on an equilibrium from the low $q_{95}$ discharge 31128 at $3500 \mathrm{~ms}$, with the $n=2$ coil configuration. Shown in blue are the harmonics $m=1-4$, and in red all harmonics with $m>10$. Vertical dashed lines indicate the radial location of rational surfaces. 
mid-plane $\chi=0$ and near the X-point (more precisely the local maximum near the X-point), and further denoted by $\xi_{M}$ and $\xi_{X}$, respectively. These two figures of merit quantify the 3D distortion of the plasma surface due to the RMP fields. Note that for a single $n$ RMP field, the amplitude of the displacement does not vary along the toroidal angle $\phi$ - the displacement itself varies as $\xi_{n} \exp (-i n \phi)$. The third set again consists of two quantities, measuring the internal displacement of the plasma. One is the amplitude of the core kink response $A_{\text {kink }}$, defined as the peak amplitude of all poloidal Fourier harmonics in the core region $\psi_{p} \in[0,0.5]$, for the radial displacement $\xi^{1}$. The other is the edge peeling-tearing response amplitude $A_{\text {peel }}$, defined in a similar way but for the edge region $\psi_{p} \in[0.8,1]$. In reality, these two quantities show the amplitude of the low- $m$ core kink components and the high- $m$ edge peeling component, as those shown in Fig. 7. Finally, the fourth set of the figures of merit are the ratios $\xi_{X} / \xi_{M}$ and $A_{\text {peel }} / A_{\text {kink. }}$. We point out that the above quantification of the core kink versus edge peeling-tearing response is certainly not unique. In fact we have tried different ways of defining the figures of merit associated with these two types of response, and eventual findings are not sensitive to that.

The above defined quantities are plotted in Figs. 8 and 9, assuming the weak and the strong parallel SWD models, respectively, for one of the low- $q$ ASDEX Upgrade equilibria, where we vary the toroidal phasing $\Delta \Phi$. The results at $\Delta \Phi=0^{\circ}, 90^{\circ}$ and $180^{\circ}$ correspond to that shown in Figs. 6 and 7. Note that figures 8(a) and 9(a) also compare the plasma response field with the vacuum field.

Several interesting observations can be made from this quantitative comparison of computational results. Firstly and generally, the plasma response is sensitive to the toroidal phasing between the upper and lower rows of coils. In other words, the response is sensitive to the variation of the poloidal spectrum of the applied 3D RMP field. Secondly, Figs. 8(a) and 9(a) show that the resistive plasma response significantly modifies the pitch resonant radial field component near the plasma edge, compared to that of the vacuum field. In fact the vacuum field amplitude can be either reduced or even amplified, depending on the toroidal phasing. There is an about $60^{\circ}$ phase shift between the vacuum predicted maximal pitch resonant amplitude, and that predicted by the resistive response model [12]. Thirdly, the variation of the plasma displacement versus $\Delta \Phi$ depends on where the displacement is measured - at the midplane or near the X-point, in the plasma core region or near the plasma edge. Despite of this diversity, a correlation holds between the core kink (edge peeling-tearing) response and the outboard mid-plane (X-point) displacement peaking, similar to that has been computationally found for MAST plasmas [10]. As will be shown later on, this correlation seems to be robust for all low $-q$ discharges with the $n=2$ RMP configuration. The even parity coil configuration maximizes the ratio of the edge peeling-tearing response to the core kink response, as well as the ratio of the X-point displacement to the outboard mid-plane displacement, as shown in Figs. 8(d) and 9(d). A final quantitative observation is that the parallel SWD model only affects the amplitude of the plasma core response, as shown in Figs. 8(c) and 9(c). The $\Delta \Phi$ location of the peak (or minimum) values are not affected by the damping model.

Since the edge $q$ value is sensitive to the truncation of flux surfaces near the separatrix, or the smoothing of the plasma boundary near the X-point (c.f. Fig. 1), the last pitch resonant harmonic can be rather different even for two equilibria with similar $q_{95}$ values. This raises a question of the robustness of the results associated with $b_{\mathrm{res}}^{1}$, as shown in Figs. 8(a) and 9(a), against the $q$-edge value. A systematic investigation of this numerical aspect has 

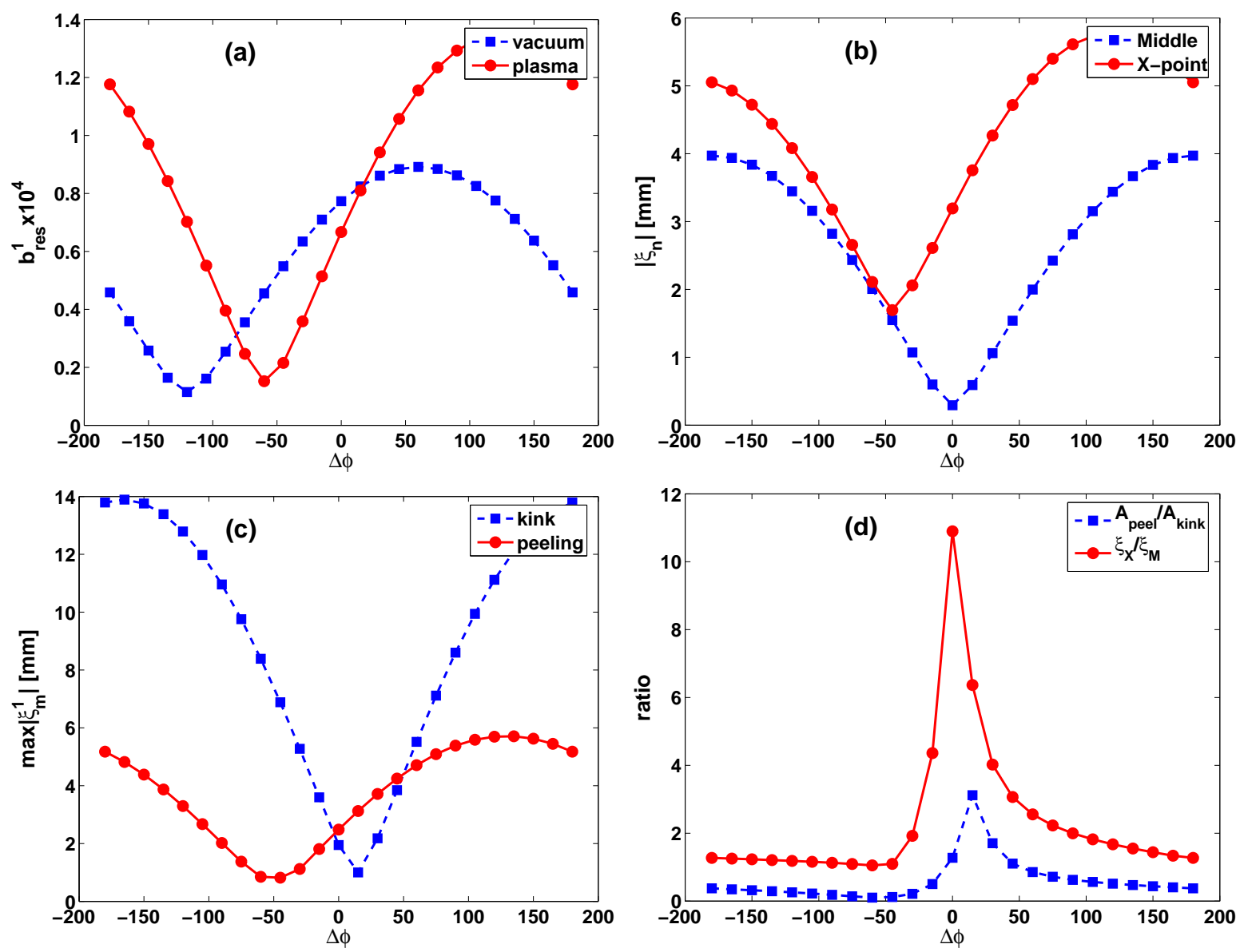

Figure 8: Comparison of various computed quantities versus the toroidal phasing $\Delta \Phi$ of the coil currents, for (a) the amplitude of the last pitch resonant radial field component between the vacuum field (dashed) and the total field including the plasma response (solid), (b) the amplitude of the plasma surface displacement at the low field side mid-plane (dashed) and near the X-point (solid), (c) the internal plasma radial displacement amplitude of the core kink (dashed) versus the edge peeling-tearing (solid) components, and (d) the ratio of the peelingtearing/kink response amplitude (dashed) versus that of the X-point/mid-plane plasma surface displacement (solid). A weak parallel sound wave damping model is used, for an equilibrium from the low $q_{95}$ discharge 31128 at $3500 \mathrm{~ms}$, with the $n=2$ coil configuration. In experiments, the strongest ELM mitigation and density pump out were observed at $\Delta \Phi 100^{\circ}$. 

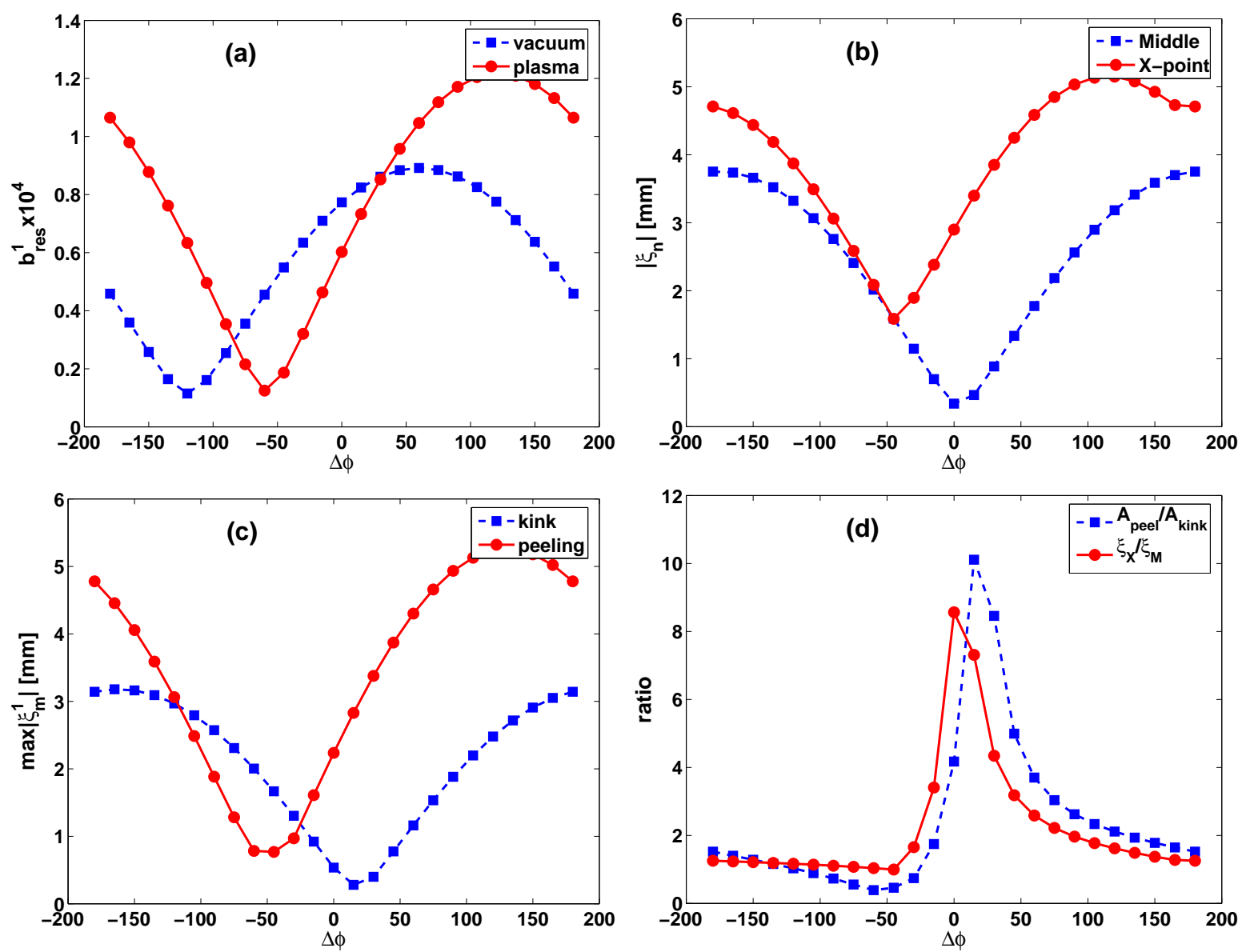

Figure 9: Comparison of various computed quantities versus the toroidal phasing $\Delta \Phi$ of the coil currents, for (a) the amplitude of the last pitch resonant radial field component between the vacuum field (dashed) and the total field including the plasma response (solid), (b) the amplitude of the plasma surface displacement at the low field side mid-plane (dashed) and near the X-point (solid), (c) the internal plasma radial displacement amplitude of the core kink (dashed) versus the edge peeling-tearing (solid) components, and (d) the ratio of the peelingtearing/kink response amplitude (dashed) versus that of the X-point/mid-plane plasma surface displacement (solid). A strong parallel sound wave damping model is used, for an equilibrium from the low $q_{95}$ discharge 31128 at $3500 \mathrm{~ms}$, with the $n=2$ coil configuration. In experiments, the strongest ELM mitigation and density pump out were observed at $\Delta \Phi 100^{\circ}$. 


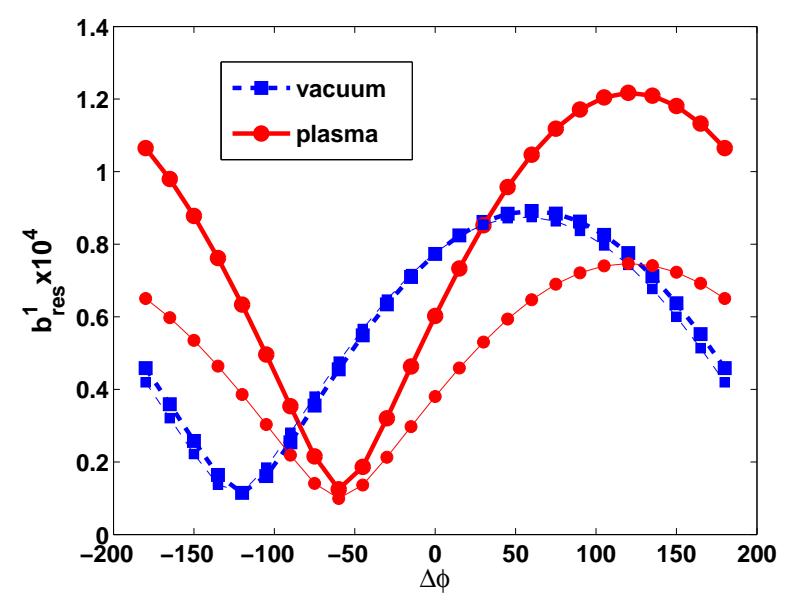

Figure 10: Comparison of the amplitude of the last pitch resonant radial field component between the vacuum field (dashed) and the total field including the plasma response (solid), for the low $q_{95}$ plasmas from discharge 31128 at 3500ms (thick lines) and from discharge 30835 at $3200 \mathrm{~ms}$ (thin lines), with the $n=2$ coil configuration. A strong parallel sound wave damping model is assumed for both plasmas.

been performed in Ref. [12], confirming the robustness of the response results. Figure 10 demonstrates this robustness by comparing the $b_{\text {res }}^{1}$ plots for two ASDEX equilibria, which have similar $q_{95}$ but different highest resonant harmonics - $m / n=12 / 2$ and $m / n=10 / 2$ for the discharges 31128 and 30835, respectively. The vacuum field, as expected, is similar. The plasma response leads to different amplitude of the last pitch resonant field component (due to differences in various plasma edge parameters such as the resistivity, the flow speed etc.). However, the optimal $\Delta \Phi$, that maximizes plasma response $b_{\text {res }}^{1}$, is about $120^{\circ}$ for both cases. This gives the same $60^{\circ}$ phase shift relative to the optimal vacuum phasing. A comprehensive study of this robustness issue will be summarized in a later Table.

This $60^{\circ}$ phase shift yields similar $b_{\text {res }}^{1}$ values between the $90^{\circ}$ coil phasing and the odd parity configuration $\left(180^{\circ}\right.$ phasing) for the total response field, whilst the $b_{\text {res }}^{1}$ values of the vacuum field are rather different. In experiments very similar results, on both the density pump-out and the RMP mitigation, were obtained for these two coil configurations [32], favouring the plasma response model.

We point out another interesting numerical observation, which is valid as shown in Figs. 8 and 9 , and generally valid also for all other plasma-coil configurations shown in this work. Namely the three figures of merit, $b_{\text {res }}^{1}, \xi_{X}$, and $A_{\text {peel }}$, defined for the total plasma response, all have a similar dependence on $\Delta \Phi$. This indicates that, if one of these three quantities can be used for interpreting the experimental results, the other two will equally apply. Indeed as shown in Ref. [32], the quantity $b_{\text {res }}^{1}$ correlates well with the change of the ELM frequency experimentally observed in a continuous $\Delta \Phi \in\left[-100^{\circ},+100^{\circ}\right]$ scan experiment, for a discharge similar to that of 30835 shown in Fig. 10. Consequently, similar correlations can be made between the quantities $\xi_{X}$ and $A_{\text {peel }}$ with the experimental results. We also note that no such correlation, in terms of the toroidal phasing $\Delta \Phi$, holds between modelling and experiments, if other quatities, such as the $b_{\text {res }}^{1}$ value of the vacuum field, $\xi_{M}$, or $A_{\text {kink }}$ are used. 


\section{4 $n=2$ plasma response at different $q_{95}$}

The plasma response described so far are obtained for the low- $q_{95}(\sim 3.8)$ ASDEX Upgrade plasmas. These results are compared with that for the high- $q_{95}(\sim 5.5)$ discharges to be reported below.

In experiments, the high- $q_{95}$ discharges are realized either by increasing the toroidal field or reducing the plasma current, while keeping the other plasma parameters similar to that of the low- $q_{95}$ discharges, see Tab. 1 . We have modelled both types of discharges, with the overall $\Delta \Phi$ phase scan results plotted in Figs. 11 and 12, respectively.

The computed results for the high- $q_{95}$, high- $B_{T}$ case, summarized in Fig. 11, show qualitatively different plasma response than the low- $q_{95}$ case, in several aspects. Firstly, the core kink amplification is greatly suppressed, as evident from Fig. 11(c). Secondly, the optimal toroidal phasing $\Delta \Phi$, that drives the maximal plasma response, in terms of $b_{\text {res }}^{1}, \xi_{X}, A_{\text {peel }}$, is around $-100^{\circ}$. For the low $-q_{95}$ plasmas, this optimal value is around $+130^{\circ}$. Finally and perhaps more interestingly, the phase difference in $\Delta \Phi$, between that maximizing the vacuum pitchresonant field and that maximizing the total response pitch-resonant field, becomes $90^{\circ}$ for the high- $q_{95}$ case, instead of $60^{\circ}$ for the low- $q_{95}$ case shown in the previous subsection. These all points to a conclusion that the optimal coil phasing, that works for the low- $q_{95}$ discharges in terms of ELM control, may not be the optimal for the high- $q_{95}$ discharges.

In the corresponding high- $q_{95}$ experiments, the observed density pump out and ELM mitigation seems to occur for $\Delta \Phi$ ranging between $-180^{\circ}$ and $-100^{\circ}$ [32]. This is the range where the computed three figures of merit $b_{\text {res }}^{1}, \xi_{X}, A_{\text {peel }}$ reach their maxima.

The plasma response for the high- $q_{95}$, low- $B_{T}$ case, summarized in Fig. 12 , are in many aspects similar to that of the high- $q_{95}$, high- $B_{T}$ case. In particular, the same $90^{\circ}$ phase shift is observed between the vacuum and the plasma response resonant field component $b_{\text {res. }}^{1}$. The slight difference is that the optimal $\Delta \Phi$, that maximizes the X-point peaking, covers a somewhat wider range. The corresponding RMP experiments seem to indicate that the strongest ELM mitigation occurs at $\Delta \Phi \sim-160^{\circ}$ [32].

A peculiar feature of the high- $q_{95}$ plasma response is reported in Fig. 13(a), where we show the plasma radial distribution of the plasma radial displacement. We find that the largest displacement among what we call the edge peeling-tearing response is for the $m / n=11 / 2$ harmonic. This is different from the typical edge response as those shown in Fig. 7. We figure out that this large 11/2 displacement is associated with the local flattening of the $q$-profile near the $q=5.5$ rational surface. This local flattening is caused by the large bootstrap current generated in the pedestal region for this discharge. Further study need to be carried out, in order to understand whether this peculiar response can be related to experimental observations.

\subsection{Plasma response to $n=4$ coil current}

The plasma response is also computed assuming the $n=4$ RMP coil configuration, based on an equilibrium from the low- $q_{95}$ discharge 31128 at $3500 \mathrm{~ms}$. In experiments, only even or 

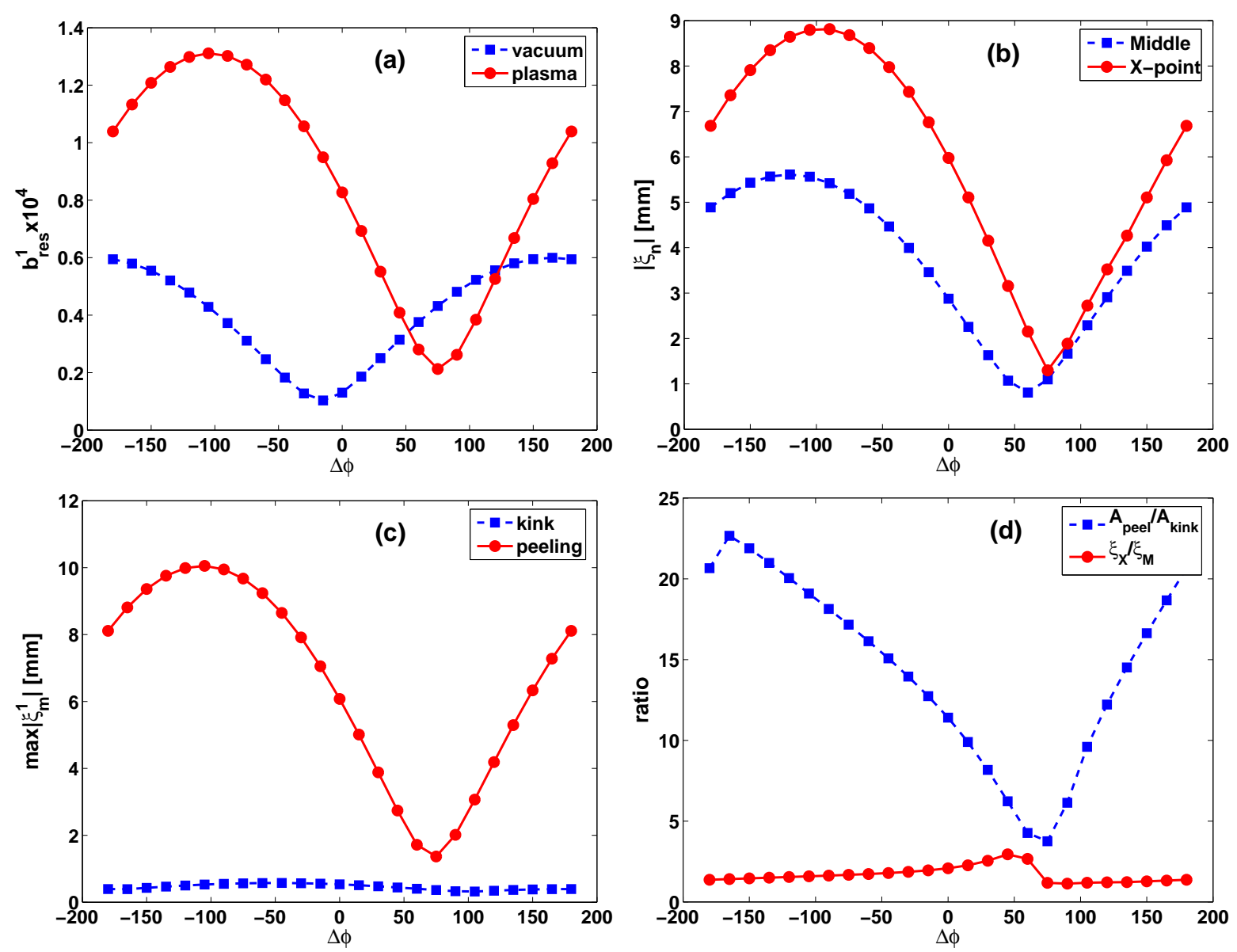

Figure 11: Comparison of various computed quantities versus the toroidal phasing $\Delta \Phi$ of the coil currents, for (a) the amplitude of the last pitch resonant radial field component between the vacuum field (dashed) and the total field including the plasma response (solid), (b) the amplitude of the plasma surface displacement at the low field side mid-plane (dashed) and near the X-point (solid), (c) the internal plasma radial displacement amplitude of the core kink (dashed) versus the edge peeling-tearing (solid) components, and (d) the ratio of the peelingtearing/kink response amplitude (dashed) versus that of the X-point/mid-plane plasma surface displacement (solid). A strong parallel sound wave damping model is used, for an equilibrium from the high $q_{95}$ high $B_{T}$ discharge 30684 at $4005 \mathrm{~ms}$, with the $n=2$ coil configuration. In experiments, the strongest ELM mitigation and density pump out were observed at $\Delta \Phi-100^{\circ}$. 

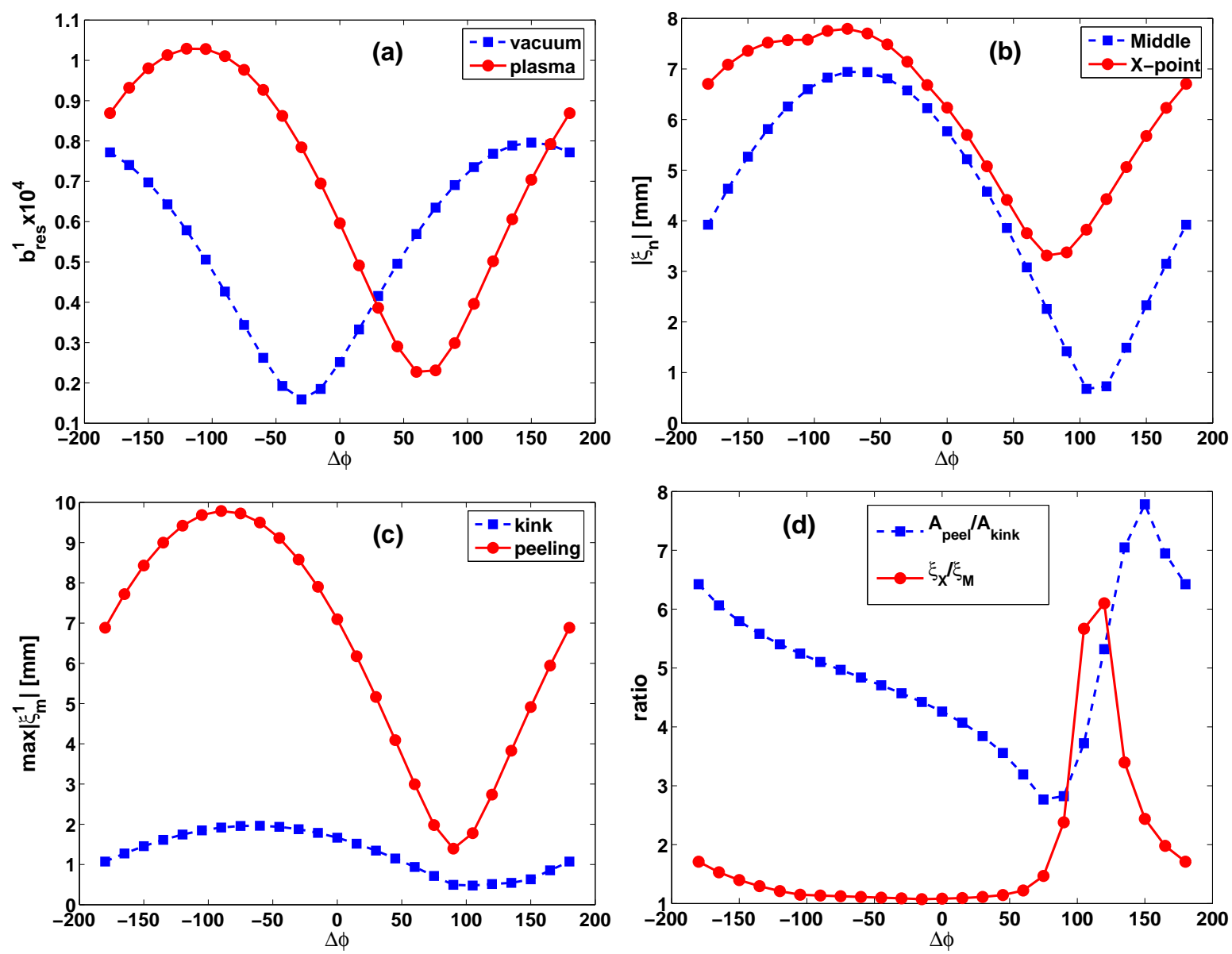

Figure 12: Comparison of various computed quantities versus the toroidal phasing $\Delta \Phi$ of the coil currents, for (a) the amplitude of the last pitch resonant radial field component between the vacuum field (dashed) and the total field including the plasma response (solid), (b) the amplitude of the plasma surface displacement at the low field side mid-plane (dashed) and near the X-point (solid), (c) the internal plasma radial displacement amplitude of the core kink (dashed) versus the edge peeling-tearing (solid) components, and (d) the ratio of the peelingtearing/kink response amplitude (dashed) versus that of the X-point/mid-plane plasma surface displacement (solid). A strong parallel sound wave damping model is used, for an equilibrium from the high $q_{95}$ low $B_{T}$ discharge 31131 at $6400 \mathrm{~ms}$, with the $n=2$ coil configuration. In experiments, the strongest ELM mitigation and density pump out were observed at $\Delta \Phi-100^{\circ}$. 

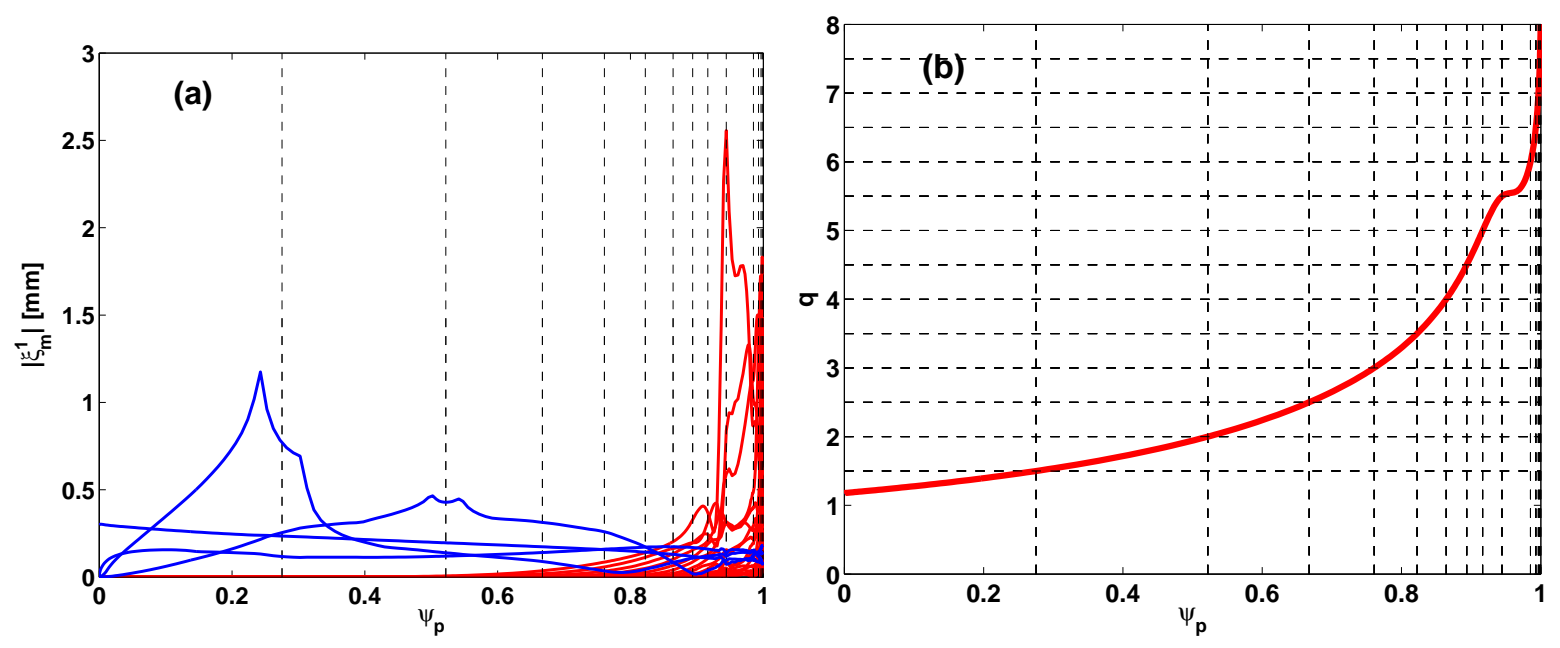

Figure 13: Correlation between (a) a large $m / n=11 / 2$ radial field response, and (b) the local flattening of the safety factor near the $q=11 / 2$ rational surface, for the high $q_{95}$ plasma from discharge 30684 at $4005 \mathrm{~ms}$, with the $n=2$ coil configuration. Vertical dashed lines indicate the radial location of rational surfaces.

odd parity is possible due to the finite number (eight) of coils along the toroidal angle. In the MARS-F modelling, however, we can still continuously scan the toroidal phasing $\Delta \Phi$ between the upper and lower rows. This is possible because we consider an analytic dependence of the coils current $\propto \exp (-i n \phi)$, with a single $n$, along the toroidal angle. This effectively assumes an infinite number of coils along the toroidal angle.

Figure 14 summarizes the results from such a $\Delta \Phi$ scan, again for various figures of merit as defined before, quantifying the plasma response. Compared to the $n=2$ response shown in Fig. 9 (the same plasma equilibrium with the same SWD assumption), there are certain similarities and certain differences.

For the $b_{\text {res }}^{1}$ field response, the optimal $\Delta \Phi$ values maximizing the vacuum and the total response field are both shifted by about $-45^{\circ}$, compared to the $n=2$ case. Thus resulting in the same $60^{\circ}$ phase difference between the vacuum field peaking and the response field peaking. In terms of the plasma surface displacement, the X-point displacement peaks at $\Delta \Phi \simeq 120^{\circ}$, similar to that shown in Fig. 9(b) for the $n=2$ configuration. However, the outboard mid-plane peaking occurs at different $\Delta \Phi$. The internal plasma displacement is rather different between $n=2$ and $n=4$. In particular, the core kink response almost diminishes in the $n=4$ response, for all toroidal phasing. This is different from the low- $q_{95}, n=2$ response, but similar to the high- $q_{95}, n=2$ response. The toroidal phasing, that maximizes the ratio of the X-point to the mid-plane displacement amplitude, occurs at $\Delta \Phi \simeq 75^{\circ}$ for the $n=4$ response, compared to the $\Delta \Phi \simeq 0^{\circ}$ optimal phasing (even parity) for the corresponding low- $q_{95}, n=2$ response shown in Fig. 9(d).

Because the core kink component is small with the $n=4$ coil configuration, the computed plasma response is overall insensitive to the parallel SWD physics, including the plasma surface displacement shown in Fig. 15. Here we also compare the computed plasma surface displacement between the odd and even parity cases, with experimentally realizable coil configurations. No significant difference is found between these two cases, in terms of the plasma 

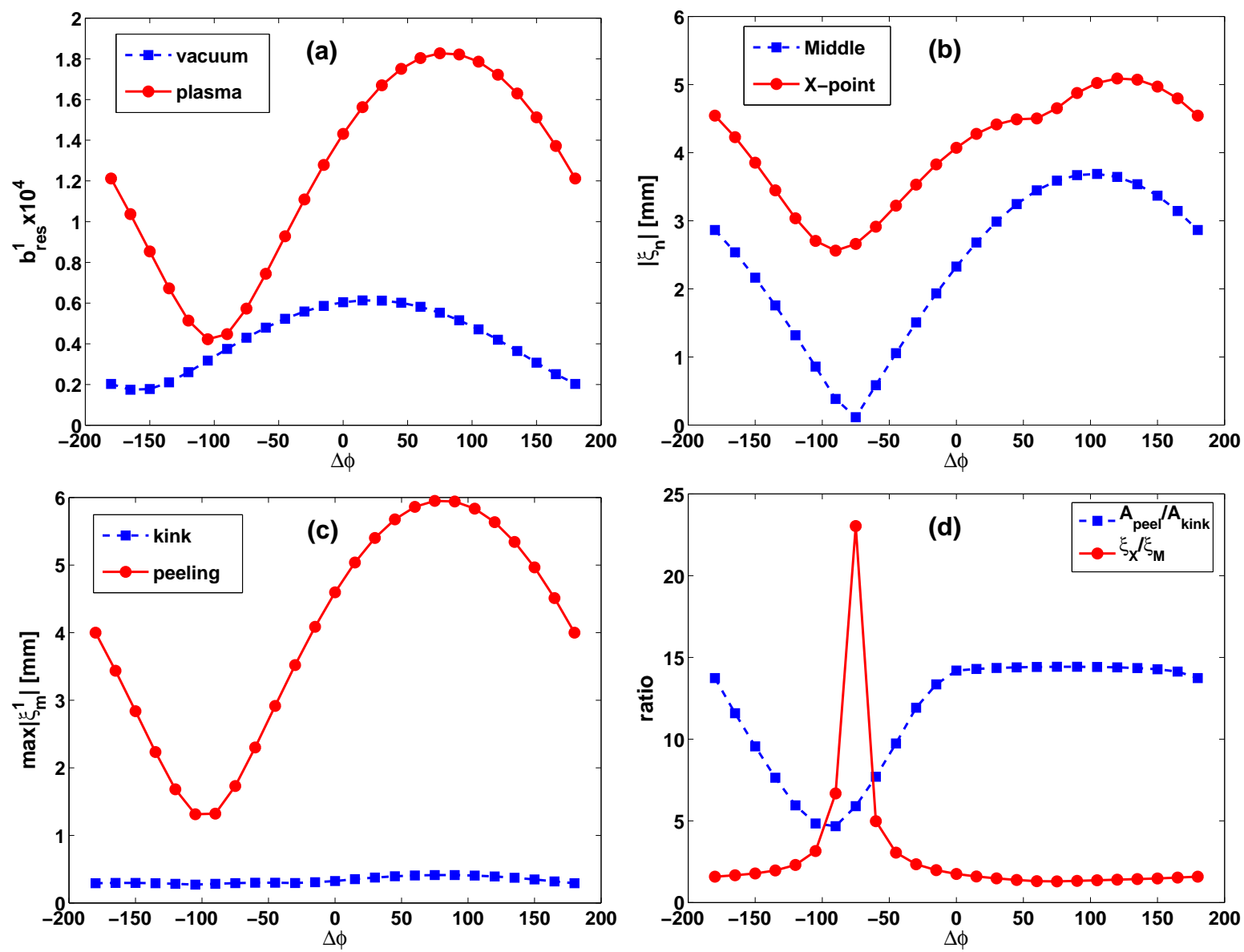

Figure 14: Comparison of various computed quantities versus the toroidal phasing $\Delta \Phi$ of the coil currents, for (a) the amplitude of the last pitch resonant radial field component between the vacuum field (dashed) and the total field including the plasma response (solid), (b) the amplitude of the plasma surface displacement at the low field side mid-plane (dashed) and near the X-point (solid), (c) the internal plasma radial displacement amplitude of the core kink (dashed) versus the edge peeling-tearing (solid) components, and (d) the ratio of the peelingtearing/kink response amplitude (dashed) versus that of the X-point/mid-plane plasma surface displacement (solid). A strong parallel sound wave damping model is used, for an equilibrium from the low $q_{95}$ discharge 31128 at $3500 \mathrm{~ms}$, with the $n=4$ coil configuration. 

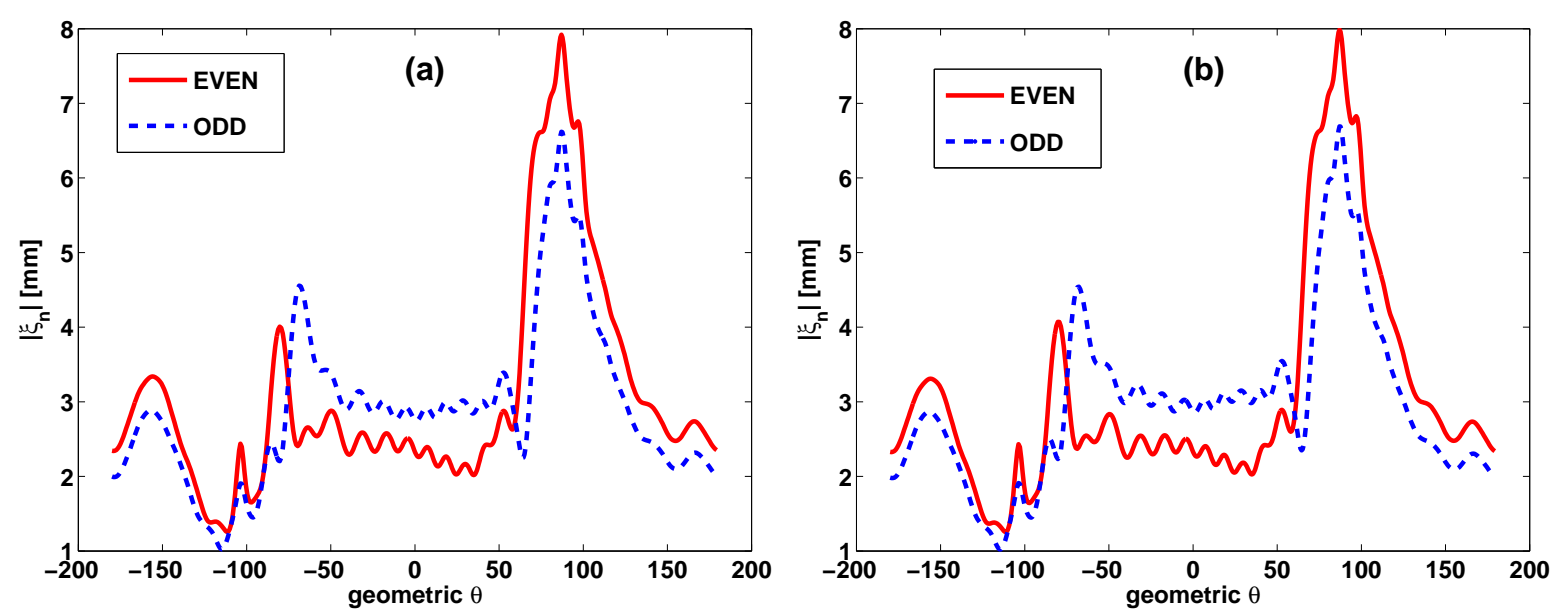

Figure 15: Comparison of the amplitude of the compute $n=4$ plasma surface displacement versus the geometric poloidal angle, between the odd (dashed) and even (solid) parity coil configurations, assuming (a) a weak, and (b) a strong parallel sound wave damping, based on the low $q_{95}$ discharge 31128 at $3500 \mathrm{~ms}$ with the $n=4$ coil configuration.

displacement. The peak displacement is computed to be about $8 \mathrm{~mm}$ with $6.5 \mathrm{kAt}$ coil current.

For the purpose of interpreting the experimental results in ASDEX Upgrade, in particular in the toroidal phasing scan RMP experiments, in Tab. 2 we list all the $\Delta \Phi$ values that maximize the MARS-F computed figures of merit as defined in this Section, for all the discharges modelled in this work. In the Table, $b_{v}^{1}$ and $b_{p}^{1}$ denote the $\Delta \Phi$ values for the peak amplitude of pitch resonant harmonic $b_{\text {res }}^{1}$ nearest to the plasma surface, for the vacuum and the plasma response field, respectively. $b_{p v}^{1}$ denotes the phase difference between $b_{p}^{1}$ and $b_{v}^{1} . \xi_{M}$ and $\xi_{X}$ denote the $\Delta \Phi$ values for the peak amplitude of the plasma surface displacement, at the outboard mid-plane and near the X-point, respectively. $\xi_{X} / \xi_{M}$ denotes the $\Delta \Phi$ value that maximizes the ratio of the X-point displacement to that of the mid-plane. $A_{k k}$ and $A_{p l}$ denote the $\Delta \Phi$ values for the peak amplitude of the core kink versus the edge peeling-tearing radial displacement, respectively. Finally, $A_{p l} / A_{k k}$ denotes the $\Delta \Phi$ value that maximizes the ratio of the edge peeling-tearing displacement to that of the core kink component.

Firstly we point out that not all the data associated with the core kink component have practical relevance, since for some cases, e.g. the $n=4$ configuration or the low- $q_{95} n=2$ configuration, the core kink response nearly diminishes for all toroidal phasing. The second comment is that the optimal $\Delta \Phi$ values are approximate - the toroidal phasing scans have been performed with $15^{\circ}$ interval in the $\Delta \Phi$ value.

With the above comments, we extract certain common features from the computed database presented in Tab. 2. (i) The optimal phasing is generally not sensitive to the parallel SWD physics that we assumed in the modelling. (ii) There is generally a $60^{\circ}$ phase shift between pitch-resonant vacuum field component and that of the total response field in the plasma edge region, for the low- $q_{95}$ discharges. This $60^{\circ}$ phase shift is in contrast with the $90^{\circ}$ phase shift computed for the high- $q_{95}$ discharges. (iii) In all the low- $q_{95} n=2$ cases, the largest ratio of the X-point plasma surface displacement to outboard mid-plane displacement occurs near the even parity coil configuration. In correlation with this, the ratio of the edge peeling-tearing 
Table 2: Toroidal phase $\Delta \Phi$ for peak amplitude of various quantities associated with the plasma response. $\kappa_{\|}$is the numerically assumed strength of the parallel sound wave damping.

\begin{tabular}{|r|r|r|r|r|r|r|r|r|r|r|r|r|}
\hline Shot\# & $\mathrm{T}(\mathrm{ms})$ & $\mathrm{n}$ & $\kappa_{\|}$ & $b_{v}^{1}$ & $b_{p}^{1}$ & $b_{p v}^{1}$ & $\xi_{M}$ & $\xi_{X}$ & $\xi_{X} / \xi_{M}$ & $A_{k k}$ & $A_{p l}$ & $A_{p l} / A_{k k}$ \\
\hline \hline 30835 & 3200 & 2 & 0.1 & 30 & 90 & 60 & -165 & 90 & 15 & 180 & 105 & 0 \\
\hline 30835 & 3200 & 2 & 1.5 & 30 & 90 & 60 & -165 & 180 & 15 & 180 & 120 & 0 \\
\hline 31128 & 3500 & 2 & 0.1 & 60 & 120 & 60 & 180 & 120 & 0 & -165 & 135 & 15 \\
\hline 31128 & 3500 & 2 & 1.5 & 60 & 120 & 60 & 180 & 120 & 0 & -165 & 135 & 15 \\
\hline 31128 & 3500 & 4 & 1.5 & 15 & 75 & 60 & 105 & 120 & -75 & 75 & 75 & 75 \\
\hline 30684 & 4005 & 2 & 0.1 & 165 & -105 & 90 & -135 & -105 & 45 & -75 & -105 & -150 \\
\hline 30684 & 4005 & 2 & 1.5 & 165 & -105 & 90 & -120 & -90 & 45 & -45 & -105 & -165 \\
\hline 30684 & 6950 & 2 & 0.1 & 165 & -105 & 90 & -150 & -105 & 30 & 135 & -105 & -90 \\
\hline 30684 & 6950 & 2 & 1.5 & 165 & -105 & 90 & -120 & -105 & 45 & -60 & -105 & -150 \\
\hline 31131 & 6400 & 2 & 1.5 & 150 & -120 & 90 & -75 & -75 & 120 & -60 & -90 & 150 \\
\hline
\end{tabular}

response amplitude to that of the core kink response also peaks near even parity. Such a correlation does not generally hold for either high- $q_{95}$ or the $n=4$ coils, largely due to the fact that the core kink response does not appear.

\section{Toroidal torques}

We also compute various toroidal torque components associated with the plasma response to the 3D RMP fields. These torque components give a good indication for the potential flow damping due to these 3D fields, but do not quantitatively inform the amount of toroidal flow speed that will eventually be reduced. This is because only the linear plasma response is used in computing these torques. A more quantitative modelling of the flow damping would require solving an initial value problem involving non-linear coupling between the $n \neq 0$ plasma response and the $n=0$ toroidal momentum balance condition $[17,16,18]$, which is out of the scope of the present study.

Three torque components are computed based on the linear plasma response: the resonant electromagnetic torque, the neoclassical toroidal viscous (NTV) torque, as well as the toroidal torque associated with the Reynolds stress. The toroidal component of the net resonant electromagnetic torque (in $[\mathrm{Nm}]$ ) is computed as

$$
T_{j \times b}^{\mathrm{net}}=\operatorname{Re} \int R^{2} \nabla \phi \cdot \mathbf{j} \times \mathbf{b}^{*} d V_{p}=4 \pi^{2} R_{0}^{3} \int_{0}^{1} T_{j \times b} d s,
$$

where $\mathbf{j}$ and $\mathbf{b}$ are the computed perturbed current and magnetic field, respectively, resulted from the plasma response to the RMP field. The notation * indicates complex conjugate. The net torque is computed in the whole plasma volume $V_{p}$. The surface averaged torque density $T_{j \times b}$ (in $\left[\mathrm{N} / \mathrm{m}^{2}\right]$ ) is also defined according to the above expression (3). Similarly, the toroidal torque due to the Reynolds stress is computed as

$$
T_{\mathrm{REY}}^{\mathrm{net}}=-\operatorname{Re} \int R^{2} \nabla \phi \cdot(\rho \mathbf{v} \cdot \nabla) \mathbf{v}^{*} d V_{p}=4 \pi^{2} R_{0}^{3} \int_{0}^{1} T_{\mathrm{REY}} d s
$$




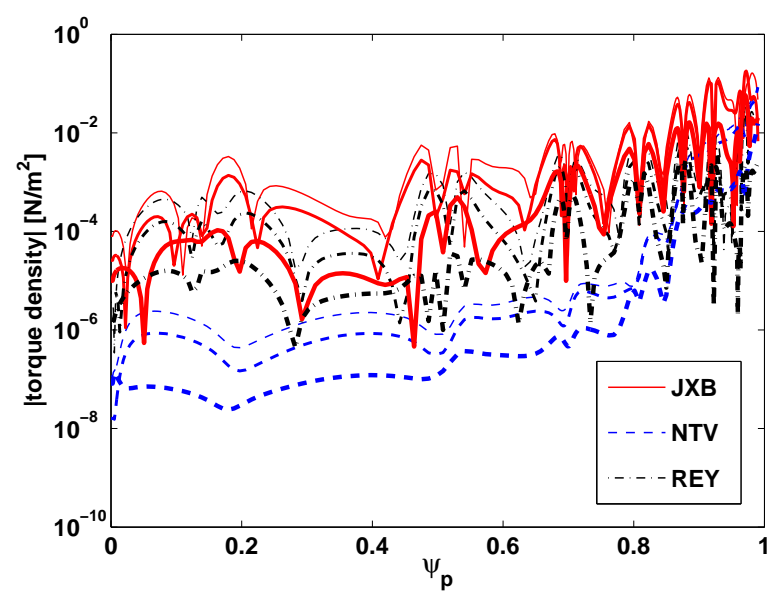

Figure 16: Comparison of various toroidal torque densities - the resonant electromagnetic torque (JXB), the neoclassical viscous torque (NTV), and the torque due to the Reynolds stress (REY) - computed from the linear plasma response, for the low $q_{95}$ discharge 31128 at $3500 \mathrm{~ms}$ with the $n=2$ coil configuration. The decreasing thickness of the lines denotes $\Delta \Phi=0^{\circ}, 90^{\circ}$, and $180^{\circ}$, respectively.

where $\rho$ is the equilibrium density of the plasma, $\mathbf{v}$ the perturbed velocity due to the RMP response. The NTV torque in this work is mainly computed using formulas from Ref. [43], where various NTV regimes are smoothly connected.

Figure 16 compares the radial distributions of the torque densities for all three components. The computations are performed for a low $q_{95}$ discharge with the $n=2$ coil configuration. Results with three choices of coil phasing $\left(\Delta \Phi=0^{\circ}, 90^{\circ}\right.$, and $\left.180^{\circ}\right)$ are presented and distinguished by the thickness of the lines. For all three torque components, generally the even parity (thickest lines) coil configuration yields the weakest torque, whilst the odd parity configuration (thinnest lines) yields the strongest torque. This is particularly evident for the NTV torque in the plasma core region. This is largely correlated to the core kink response, which is the weakest at even parity coil phasing, and the strongest at the odd parity, as shown by Fig. 9(b) and (c) for the same discharge.

Figure 16 also shows that the NTV torque is relatively small compared to the other two torques, in particular in the plasma core region. Normally the NTV torque is contributed by the socalled resonant and non-resonant components, depending on the resonance condition in the particle velocity space. The resonant NTV torque occurs when the toroidal precessional drift frequency of bulk thermal particles matches the $E \times B$ flow frequency of the plasma [43]. This resonance normally leads to substantial enhancement of the NTV torque, compared to the non-resonant contribution. Since the toroidal precession of thermal particles is usually slow, the resonant NTV torque can be important only if the $E \times B$ flow is also slow. A detailed analysis of the NTV torque contributions for this case, shown in Fig. 17 for the even parity coil configuration, reveals that the NTV torque, in the bulk region of the plasma, is contributed by the non-resonant component which is small. The resonant contribution is dominant only near the magnetic axis as well as near the plasma edge. This happens because the $E \times B$ flow speed is small near the plasma edge, and the precessional drift speed of particles is relatively large near the magnetic axis (the precessional drift frequency scales inversely with the plasma 


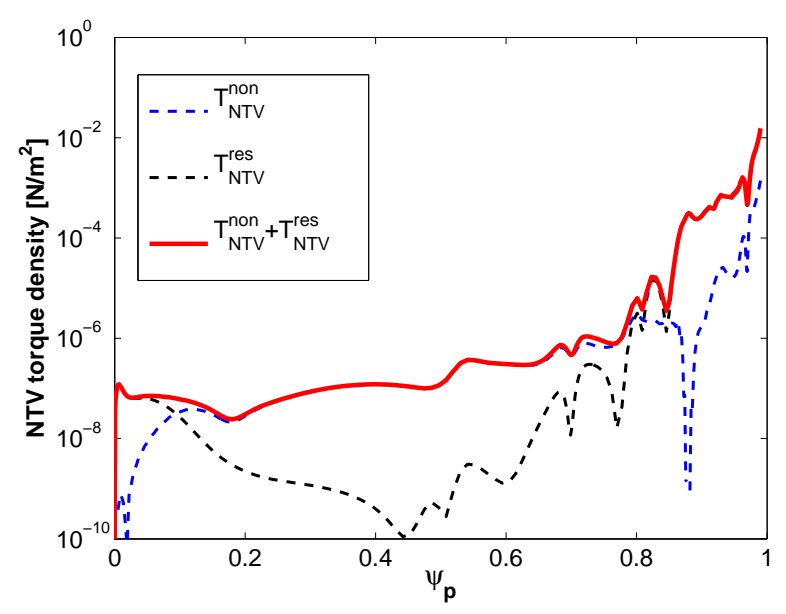

Figure 17: Comparison of resonant (in the particle velocity phase space) versus non-resonant contributions to the NTV torque density, for the low $q_{95}$ discharge 31128 at $3500 \mathrm{~ms}$ with the $n=2$ coil configuration at even parity $\left(\Delta \Phi=0^{\circ}\right)$.

minor radius according to the large aspect ratio calculations). Both factors lead to the possible satisfaction of the resonance conditions in these two regions. In the bulk plasma region, however, the $E \times B$ flow is too fast in this ASDEX Upgrade plasma to enter into the resonance regime.

Compared to the $n=2$ coil configuration, the $n=4$ configuration, with the same coil current, provides less torques (by about one order of magnitude) in the bulk plasma region, as shown in Fig. 18. On the other hand, the torque amplitudes are comparable near the plasma edge (in the pedestal region), for both $n=2$ and $n=4$ RMP coil current configurations. Another striking difference between these two configurations is that for the $n=4$ configuration, the torques are of similar magnitude between the odd (thin lines) and even (thick lines) parities, whilst for the $n=2$ configuration, the odd parity produces much larger torques than the even parity.

As aforementioned, the NTV torque amplitude is sensitive to the resonance conditions in the particle phase space. In order to verify this, we also performed dedicated MARS-F runs, where we keep everything the same as that in Fig. 18(b), but significantly reduce the plasma flow speed, by a factor of 5. The results are shown in Fig. 19(a). Whilst the electromagnetic $\mathbf{j} \times \mathbf{b}$ torque is not much affected by the reduction of the flow speed, the NTV torque does become substantially larger. This suggests that, at much lower plasma rotation speed (and hence the $E \times B$ flow), the NTV torque can be significantly enhanced by the resonant contribution.

Another interesting aspect is that Shaing's semi-analytic NTV model [43], which is what we have adopted in majority of the present modelling, usually underestimates the torque amplitude compared to the full toroidal model, as has been shown in Ref. [44]. This holds as long as the plasma collisionality is not too low to enter into the so called $v$-regime for the nonresonant NTV torque and the super-banana plateau regime for the resonant NTV torque. The MARS-K [40] computed NTV torque, based on full toroidal model and including all possible drift kinetic effects, is indeed larger than that predicted by the Shaing's model for this ASDEX Upgrade plasma, as shown by the comparison of Fig. 19(b) with Fig. 18(b). In fact in the 

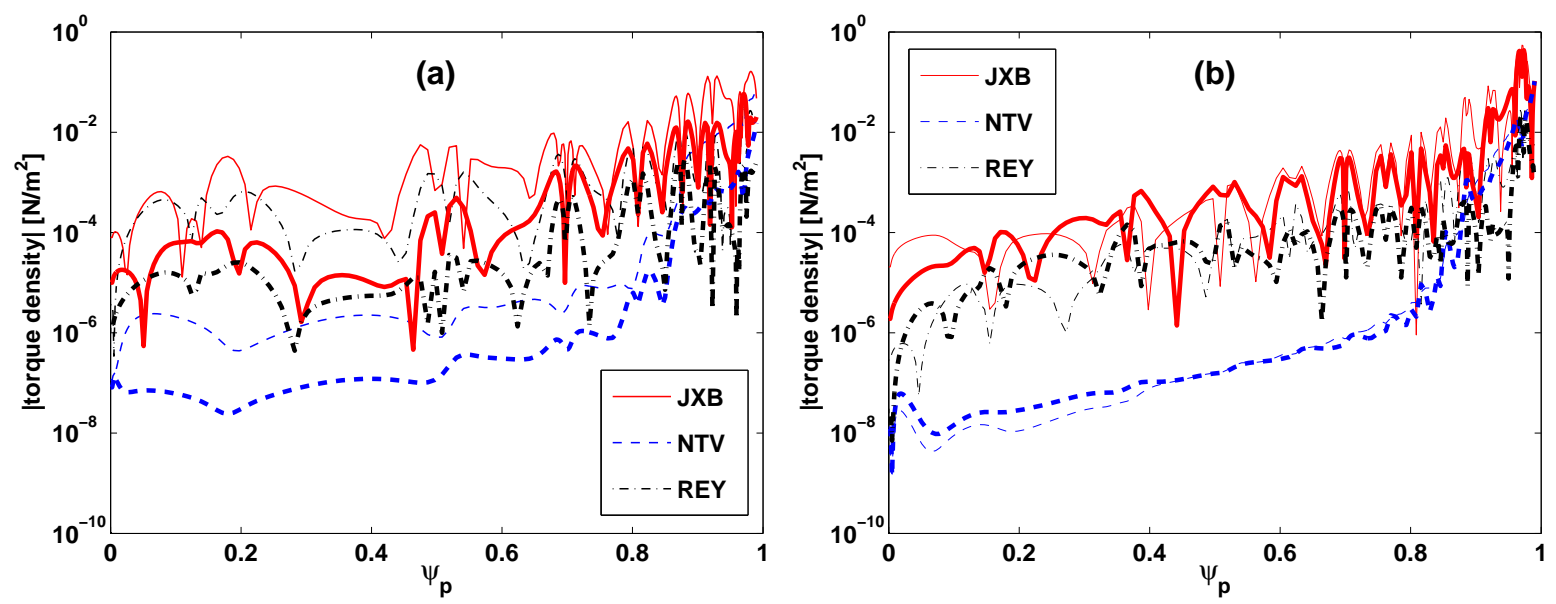

Figure 18: Comparison of various toroidal torque densities - the resonant electromagnetic torque (JXB), the neoclassical viscous torque (NTV), and the torque due to the Reynolds stress (REY) - computed from the linear plasma response for the low $q_{95}$ discharge 31128 at $3500 \mathrm{~ms}$ with (a) the $n=2$, and (b) the $n=4$ coil configurations. Either odd (thin lines) or even (thick lines) parity of the coil current is assumed.

plasma core region, the full NTV model predicts larger torque provided by the NTV physics, than that due to the resonant electromagnetic Maxwell stress and due to the Reynolds stress.

A more quantitative comparison can be made by evaluating the net torque by integrating the torque density over certain plasma region of the plasma minor radius. Table 3 summarizes the findings for representative discharges, for both $n=2$ and $n=4$ coil configurations with various toroidal phasing. The net torques are evaluated either over the whole plasma column (up to $99 \%$ of the equilibrium poloidal flux), or (essentially) over the pedestal region. The total net torques, as well as the three individual contributions (electromagnetic, NTV, Reynolds), are presented in the Table.

For the low $q_{95}$ discharge 31128 , the total net torque essentially comes from the plasma edge region. This holds well for three torque components. Whilst the NTV torque is either larger than the $\mathbf{j} \times \mathbf{b}$ torque, or dominant over all other two components, the Reynolds torque is always small by order of magnitude. We also point out that, as has been qualitatively shown by previous figures, the total net torque significantly varies depending on the coil phasing.

The torque contribution is slightly more complicated for the high $q_{95}$ discharge 31131 . The pedestal region contributes a small fraction (about 10\%) to the net electromagnetic torque, a larger fraction (over 70\%) to the net NTV torque, and nearly $100 \%$ to the Reynolds torque. In terms of the net torque over the whole plasma column, all three torque components contribute comparable amount. The total net torque is comparable also between the odd and even parity coil configurations. The computed torques can be useful in interpreting the measured flow damping during the RMP experiments. A systematic analysis of the experimental data and the comparison with the modelling results will be part of a future work. 

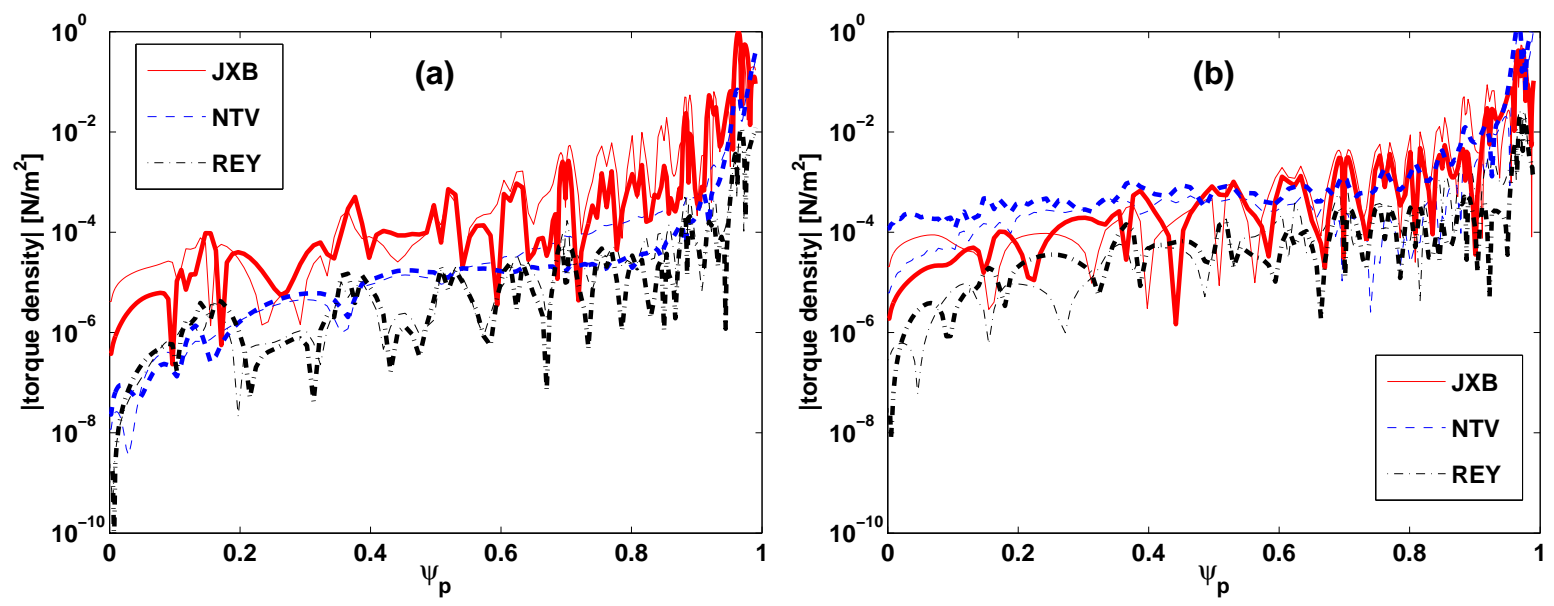

Figure 19: Comparison of various toroidal torque densities - the resonant electromagnetic torque (JXB), the neoclassical viscous torque (NTV), and the torque due to the Reynolds stress (REY) - computed from the linear plasma response, for the low $q_{95}$ discharge 31128 at $3500 \mathrm{~ms}$ with the $n=4$ coil configuration, assuming (a) 5 times reduced plasma flow speed compared with the experiment, and (b) the full flow speed and a full drift kinetic model for the NTV torque computation. Either odd (thin lines) or even (thick lines) parity of the coil current is assumed.

Table 3: Net torques $(\mathrm{Nm})$ integrated over $\psi_{p} \in[0,0.99]$ ('full') and over $\psi_{p} \in[0.9,0.99]$. Strong parallel SWD assumed.

\begin{tabular}{|c|c|c|r|r|r|r|r|r|r|r|c|}
\hline shot\# & \multicolumn{1}{|c|}{$\mathrm{ms}$} & $n$ & $\Delta \Phi$ & $T_{\mathrm{JXB}}^{\text {full }}$ & \multicolumn{1}{c|}{$T_{\mathrm{JXB}}^{\text {edge }}$} & $T_{\mathrm{NTV}}^{\text {full }}$ & $T_{\mathrm{NTV}}^{\text {edge }}$ & $T_{\mathrm{REY}}^{\text {full }}$ & $T_{\mathrm{REY}}^{\text {edge }}$ & $T_{\mathrm{TOT}}^{\text {full }}$ & $T_{\mathrm{TOT}}^{\text {edge }}$ \\
\hline \hline 31128 & 3500 & 2 & $0^{\circ}$ & -0.034 & -0.030 & -0.017 & -0.016 & -0.001 & -0.001 & -0.052 & -0.047 \\
\hline 31128 & 3500 & 2 & $180^{\circ}$ & -0.158 & -0.112 & -0.171 & -0.160 & -0.019 & -0.015 & -0.348 & -0.288 \\
\hline 31128 & 3500 & 2 & $90^{\circ}$ & -0.106 & -0.081 & -0.136 & -0.128 & -0.015 & -0.013 & -0.257 & -0.221 \\
\hline 31128 & 3500 & 4 & $0^{\circ}$ & 0.041 & 0.047 & -0.107 & -0.105 & -0.005 & -0.005 & -0.072 & -0.063 \\
\hline 31128 & 3500 & 4 & $180^{\circ}$ & -0.055 & -0.053 & -0.116 & -0.114 & -0.013 & -0.012 & -0.184 & -0.179 \\
\hline 31131 & 6400 & 2 & $0^{\circ}$ & -0.267 & 0.030 & -0.123 & -0.084 & -0.118 & -0.124 & -0.509 & -0.178 \\
\hline 31131 & 6400 & 2 & $180^{\circ}$ & -0.180 & 0.003 & -0.106 & -0.081 & -0.156 & -0.158 & -0.442 & -0.236 \\
\hline
\end{tabular}




\section{Summary and discussion}

The MARS-F/K codes have been applied to model the plasma response to the RMP fields in the ASDEX Upgrade low-collisionality ELM control experiments. A systematic investigation is carried out, taking into account various plasma and coils configurations.

For the low $q$ plasmas, with $q_{95} \sim 3.8$, the plasma response to each individual row of the Bcoil currents generates a core kink component, which is absent in the vacuum RMP field. This core kink component, occurring mainly for low $n$ RMP fields, is identified by the poloidal Fourier harmonics of the perturbed radial magnetic field. When both rows of the B-coils are powered to produce the RMP fields, depending on the toroidal phase difference between the upper and lower rows, this plasma amplified core kink component can either be cancelled or reinforced. For instance, with the $n=2$ coil configuration, even parity leads to the cancellation whilst odd parity leads to the reinforcement. The opposite occurs for the $n=1$ coil configuration. The cancellation (reinforcement) of the core kink response coincides with the cancellation (reinforcement) of the plasma surface displacement at outboard mid-plane. As a result, the core kink amplification by the plasma leads to a large mid-plane displacement, whilst the absence of the core kink (due to the cancellation) amplification leads to the plasma displacement peaking near the X-point.

The core kink response, however, is relatively weak for low $q$ plasmas with the $n=4$ coil current configuration, or for high $q$ plasmas. Nevertheless, we find a good correlation between the edge peeling (core kink) plasma response and the plasma displacement peaking near the $\mathrm{X}$-point (low field side mid-plane), for all low $q$ plasmas, similar to an earlier finding for the MAST plasmas [10].

Furthermore, the plasma response produces a phase shift, compared to the vacuum RMP field, in the optimal coil phasing $\Delta \Phi$ that maximizes the resonant magnetic perturbations (i.e. the magnetic islands) near the plasma edge. The edge resonant field components have been shown to serve as a good indicator for the ELM mitigation in both MAST and ASDEX Upgrade experiments [32]. Therefore, the knowledge of the plasma response induced phase shift is critical in designing the optimal coil phasing in the ELM control experiments. The MARS-F computed phase shift, which is also well confirmed by the experimental results, is around 60 degrees for the low $q\left(q_{95} \sim 3.8\right)$ discharges in ASDEX Upgrade, for both $n=2$ and $n=4$ coil current configurations, and around 90 degrees for the high $q\left(q_{95} \sim 5.5\right)$ discharges. The 60 degrees phase shift has previously been found [12] for a particular plasma, but is now confirmed here to be a reasonably robust quantity. We also find that the optimal coil phasing is not sensitive to the damping models (weak versus strong parallel sound wave damping) that we assume in the MARS-F runs. On the other hand, a strong sound wave damping does moderately suppress the core kink response, but with little effect on the edge peeling response.

Taking into account the plasma response, the modelling predicted optimal coil phasing that maximizes the edge resonant field amplitude, for the $n=2$ coil current configuration, is around 100 degrees for the low $q$ plasmas, and around -100 degrees for the high $q$ plasmas. These predictions are well verified in the recent ASDEX Upgrade experiments [32]. Since the variation of the X-point displacement with $\Delta \Phi$ follows well with that the edge resonant field, these optimal coil phasings also maximize the $\mathrm{X}$-point displacement. 
For low $q$ plasmas, the toroidal torque components, evaluated based on the linear plasma response, are dominant near the plasma edge (in the pedestal region). Both the resonant electromagnetic and the NTV torque provide the main contributions to the total net torque, with the Reynold torque contributing less than $10 \%$. The NTV contribution is generally small in the plasma core region, due to the lack of resonant NTV component. However, the NTV torque can be significantly larger in the core region at reduced plasma flow speed. The full toroidal NTV model, employed in the MARS-K code, also predicts somewhat larger NTV torque in the plasma core.

So far essentially all the ELM control experiments in ASDEX Upgrade low collisionality plasmas are carried out for two choices of the $q_{95}$ values (3.8 and 5.5 respectively). These choices are also followed by the modelling. Work is however ongoing, in order to map out the optimal coil phasing with continuously varying $q_{95}$, for both $n=2$ and $n=4$ coil configurations. Another topic of the future study is the self-consistent modelling of the toroidal flow damping for these ELM control experiments, using the initial value code MARS-Q [17].

\section{Acknowledgements}

This work has been carried out within the framework of the EUROfusion Consortium and has received funding from the Euratom research and training programme 2014-2018 under grant agreement No 633053 and from the RCUK Energy Programme [grant number EP/I501045]. Work is also part funded by National Natural Science Foundation of China (NSFC) [grant numbers 11428512 and 11405029] and by National Magnetic Confinement Fusion Science Program under grant No. 2015GB104004. The views and opinions expressed herein do not necessarily reflect those of the European Commission.

\section{References}

[1] Loarte A. et al 2007 Nucl. Fusion 47 S203

[2] Lang P.T. et al 2004 Nucl. Fusion 44665

[3] de la Luna E. et al 2009 Proc. EPS Conference on Plasma Physics, Sofia, Bulgaria, Vol. 33E P5.174

[4] Evans T.E. et al 2006 Nat. Phys. 2419

[5] Kirk A. et al 2013 Plasma Phys. Control. Fusion 55124003

[6] Liu Y.Q. et al 2010 Phys. Plasmas 17122502

[7] Heyn M.F. et al 2008 Nucl. Fusion 48024005

[8] Becoulet M. et al 2009 Nucl. Fusion 49085011

[9] Ferraro N.M. 2012 Phys. Plasmas 19056105 
[10] Liu Y.Q. et al 2011 Nucl. Fusion 51083002

[11] Haskey S. et al 2014 Plasma Phys. Control. Fusion 56035005

[12] Ryan D. et al 2015 Plasma Phys. Control. Fusion 57095008

[13] Chapman I.T. et al 2012 Plasma Phys. Control. Fusion 54105013

[14] Chapman I.T. et al 2014 Nucl. Fusion 55013004

[15] Ham C.J. et al 2014 Phys. Plasmas 21102501

[16] Liu Y.Q. et al 2012 Plasma Phys. Control. Fusion 54124013

[17] Liu Y.Q. et al 2013 Phys. Plasmas 20042503

[18] Liu Y.Q. et al 2015 Nucl. Fusion 55063027

[19] Park J.-K. et al 2007 Phys. Rev. Lett. 99195003

[20] Hirshman S.P. et al 1983 Phys. Fluids 263553

[21] Reiman A. et al 1986 Compt. Phys. Commun. 43157

[22] Suzuki Y. et al 2006 Nucl. Fusion 46 L19

[23] Hudson S.R. et al 2012 Plasma Phys. Control. Fusion 54014005

[24] Turnbull A. et al 2013 Phys. Plasmas 20056114

[25] Cooper W.A. et al 2014 Plasma Phys. Control. Fusion 56094004

[26] Orain F. et al 2013 Phys. Plasmas 20102510

[27] Park G. et al 2010 Phys. Plasmas 17102503

[28] Wang Z.R. et al 2015 Phys. Rev. Lett. 114145005

[29] Lanctot M. et al 2010 Phys. Plasmas 17030701

[30] King J.D. et al 2015 Phys. Plasmas 22072501

[31] Liu Y.Q. et al 2000 Phys. Plasmas 73681

[32] Kirk A. et al 2015 Nucl. Fusion 55043011

[33] McCarthy P.J. et al 2011 Plasma Phys. Control. Fusion 54015010

[34] Lütjens H. et al 1996 Comput. Phys. Commun. 97219

[35] Liu Y.Q. et al 2014 Plasma Phys. Control. Fusion 56104002

[36] Hammett G.W. et al 1990 Phys. Rev. Lett. 643019

[37] Bondeson A. et al 1989 Phys. Fluids B 11431

[38] Lanctot M. et al 2011 Phys. Plasmas 18056121 
[39] Liu Y.Q. et al 2005 Nucl. Fusion 451131

[40] Liu Y.Q. et al 2008 Phys. Plasmas 15112503

[41] Kirk A. et al 2013 Nucl. Fusion 53043007

[42] Nardon E. et al 2009 Plasma Phys. Control. Fusion 51124010

[43] Shaing K.C. et al 2010 Nucl. Fusion 50025022

[44] Wang Z.R. et al 2014 Phys. Plasmas 21042502 